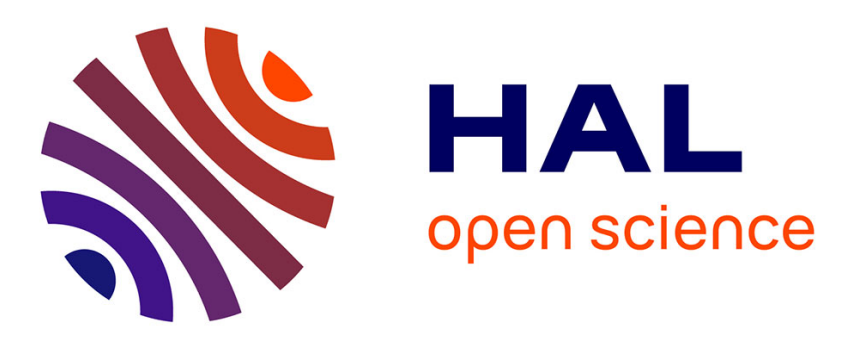

\title{
Non-Gaussian positive-definite matrix-valued random fields with constrained eigenvalues: Application to random elasticity tensors with uncertain material symmetries
}

Johann Guilleminot, Christian Soize

\section{To cite this version:}

Johann Guilleminot, Christian Soize. Non-Gaussian positive-definite matrix-valued random fields with constrained eigenvalues: Application to random elasticity tensors with uncertain material symmetries. International Journal for Numerical Methods in Engineering, 2011, 88 (11), pp.1128-1151. 10.1002/nme.3212 . hal-00684290

\section{HAL Id: hal-00684290 \\ https://hal.science/hal-00684290}

Submitted on 1 Apr 2012

HAL is a multi-disciplinary open access archive for the deposit and dissemination of scientific research documents, whether they are published or not. The documents may come from teaching and research institutions in France or abroad, or from public or private research centers.
L'archive ouverte pluridisciplinaire HAL, est destinée au dépôt et à la diffusion de documents scientifiques de niveau recherche, publiés ou non, émanant des établissements d'enseignement et de recherche français ou étrangers, des laboratoires publics ou privés. 


\title{
Non-Gaussian positive-definite matrix-valued random fields with constrained eigenvalues: application to random elasticity tensors with uncertain material symmetries*
}

\author{
J. Guilleminot, C. Soize \\ Université Paris-Est \\ Laboratoire MSME UMR8208 CNRS \\ 5 Bd Descartes, 77454 Marne la Vallée, France
}

28 APR 2011

\begin{abstract}
This paper is devoted to the construction of a class of prior stochastic models for non-Gaussian positive-definite matrix-valued random fields. The proposed class allows the variances of selected random eigenvalues to be specified and exhibits a larger number of parameters than the other classes previously derived within a nonparametric framework. Having recourse to a particular characterization of material symmetry classes, we then propose a mechanical interpretation of the constraints and subsequently show that the probabilistic model may allow prescribing higher statistical fluctuations in given directions. Such stochastic fields turn out to be especially suitable for experimental identification under material symmetry uncertainties, as well as for the development of computational multi-scale approaches where the randomness induced by fine-scale features may be taken into account. We further present a possible strategy for inverse identification, relying on the sequential solving of least-square optimization problems. An application is finally provided.
\end{abstract}

Keywords: Elasticity tensor; Material Symmetry; Maximum Entropy Principle; Probabilistic Model; Random Field; Uncertainty.

\section{INTRODUCTION}

The increasing use and modeling of heterogeneous materials gives rise to many scientific challenges defined at various scales. One of the main issues that has been extensively studied over the past fifty years concerns the analytical or computational characterization of the relationship between a complex microstructure and its overall, macroscopic behavior. Most of these contributions have been derived within a deterministic framework, considering domains that are larger than the Representative Volume Element (RVE) associated with the microstructure [17]. Quite recently, attention has been paid to the modeling of the randomness

* J. Guilleminot and C. Soize, Non-Gaussian positive-definite matrix-valued random fields with constrained eigenvalues: application to random elasticity tensors with uncertain material symmetries, International Journal for Numerical Methods in Engineering, 88(11), 1128-1151, DOI: 10.1002/nme.3212 (2011). 
induced by local microstructural features, ranging from the mesoscale modeling of random media (pioneered by Huet [10] - see also [20] and [9]- and largely discussed later on) to stochastic simulations (based on the Stochastic Finite Elements Method; see the monograph by Ghanem and Spanos [7] for instance) of structural parts made up with multi-phase materials. With reference to [10], it should be emphasized at this stage that taking into account the underlying randomness induced by microstructural features is only relevant for materials (or applications) for which the scale separation (between the RVE scale and the coarse scale - that is, the scale of either engineering application or experimental testing-) cannot be stated, yielding to the consideration of probabilistic, apparent mechanical properties. Although such a framework was initially derived for heterogeneous materials whose reinforcing constituents are characterized by a millimetric or centimetric size (such as some concretes or some fiber-reinforced composites), it may also be suitable for other classes of materials due to the constant miniaturization of systems. Clearly, the question of whether and how such mesoscopic randomness must be taken into account is application dependent and rises some theoretical issues (see for instance [18]) which are worth discussing prior to performing any probabilistic modeling on real microstructures.

While many of these previous works generally provide a quantitative computational characterization of randomness (by simulating random microstructures in relative accordance with experimental data and by computing some statistics of any response of interest; see [32] among many others), there is a need for suitable probabilistic models (that is to say, for representations of random variables that are consistent from both a mathematical and physical standpoint) allowing, for instance, experimental identification and large-scale stochastic simulations. Two kinds of approaches can then be followed.

The first one consists in modeling either the engineering modulus characterizing the random elasticity tensor with given material symmetry (e.g. the Young's modulus and Poisson ratio for the isotropic case) or equivalently, all its nonzero components. One of the main drawback of such a framework is that the construction of the probabilistic model becomes intractable for low-symmetry materials (because of the statistical dependence between the components). Indeed, it should even be pointed out that imposing a material symmetry class while considering statistical fluctuations is, in general, highly questionable, so that the joint probability distribution may always be constructed on $\mathbb{R}^{21}$ (that is to say, considering the anisotropic case). Moreover, the associated formalism does not allow fundamental properties of the elasticity tensor (such as positive-definiteness) to be taken into account easily.

Alternatively, one may consider the direct construction of a nonparametric probabilistic model for the matrix-valued random variable corresponding to a matrix representation of the stochastic elasticity tensor. Such an approach was first introduced, in the case of a tensor-valued random field and for the anisotropic class, in [25] [24]. Following a similar methodology (based on the use of the Maximum Entropy Principle; see [22] [11] [12]), Das and Ghanem derived another probabilistic model for random elasticity matrices that are deterministically bounded from below and above [4]. While these nonparametric probabilistic models benefit from both their mathematical background and minimal parametrization, they basically induce anisotropic statistical fluctuations which cannot be preferably assigned to a set of components of the random matrix. Based on this statement, Ta et al. proposed a new class (in the class of the generalized probabilistic approach of uncertainties [26]) by adding a new parameter allowing the distance to the isotropy class to be measured and partly controlled, regardless of the level of statistical fluctuations of the random elasticity matrix [30]. However, such an approach relies on the construction of a parametric probabilistic model which cannot be reasonably generalized to spaces of higher dimension.

In this work, we address the construction of a class of prior stochastic models for tensor-valued random fields. From a methodological point of view, such an issue turns out to be very important while modeling complex materials for which the microstructure cannot be described in terms of the local topology and the mechanical behavior of its constitutive phases (e.g. some biological tissues). Furthermore, it should be 
emphasized that a non-Gaussian tensor-valued random field cannot be constructed using nonparametric statistics, even if a large amount of experimental data are available (because of mathematical properties, such as symmetry, positiveness, ellipticity condition, etc., which have to be fulfilled). In addition, experimental data are often limited (in the sense that only a few realizations may be available) and correspond to partial observation, that is to say, are identified through the partial characterization of the solution of a boundary value problem (such as a displacement field measured on a part of the domain under testing). For these reasons, it is absolutely necessary that the prior stochastic model thus constructed integrates as much available mathematical information as possible in order to ensure the consistency and robustness of the model in both forward simulations and inverse identification.

This paper is organized as follows. Section 2 deals with the construction of the class of non-Gaussian positive-definite matrix-valued random fields. In particular, fundamental properties as well as strategies for the numerical Monte-Carlo simulations of random fields belonging to the proposed class are presented. Section 3 is devoted to a mechanical interpretation of the parametrization of the class. More specifically, we introduce distances in the set of elasticity tensors (from which projections onto the set of elasticity tensors with given symmetries can be defined), as well as the eigensystem characterization of material symmetries. Such definitions will be used in order to define constraints on the stochastic eigenvalues of the random elasticity tensor, so that the (mean) distance to a material symmetry class can be, to a limited extent, specified. Finally, a numerical example illustrating a possible strategy for the inverse identification of the model is presented in Section 4.

\section{PROBABILISTIC MODEL DERIVATION}

This section is devoted to the construction of the prior probabilistic model of the non-Gaussian tensorvalued random field, which will be used for modeling the apparent elasticity tensor random field at a given mesoscale. From a physical standpoint, the latter makes reference to some spatial resolution at which the apparent elasticity random tensor field is constructed and identified, that is, to the size of some window over which averaging is performed. Note at this stage that this interpretation is only conceptual, as no homogenization procedure is performed between the microscale and the considered mesoscale. When such a size is (much) smaller than the RVE size (should a RVE exist), statistical fluctuations of the elasticity field remain and have to be taken into account, thus motivating the use of the proposed derivations. Since the choice of such an averaging window naturally implies some smoothing of the fluctuations induced by finest scales, the properties of the apparent tensor random field (e.g. the spatial correlation lengths, level of fluctuations, etc.) depend on the choice of the mesoscale, but turns out to be completely independent of the geometry and of any kind of boundary condition at this mesoscale. Obviously, this mesoscale model depends on the boundary conditions at the microscale (as largely discussed in the literature), which are however not considered here since no homogenization is performed. Nevertheless, once this model is used at this spatial resolution, the identification of its parameters can be readily obtained solving the inverse stochastic problem related to the mesoscale boundary value problem.

\subsection{Overview of the construction}

Let $\mathbb{M}_{n}^{S}(\mathbb{R})$ and $\mathbb{M}_{n}^{+}(\mathbb{R}) \subset \mathbb{M}_{n}^{S}(\mathbb{R})$ be the set of all the $(n \times n)$ real symmetric and symmetric positivedefinite matrices respectively, with $n \leq 6$. Let $\mathbf{x} \mapsto[\mathbf{L}(\mathbf{x})]$ be the $\mathbb{M}_{n}^{+}(\mathbb{R})$-valued random field defined on probability space $(\Theta, \mathcal{T}, P)$, indexed by a bounded open domain $\Omega$ in $\mathbb{R}^{d}$ ( $d$ being a positive integer, $1 \leq d \leq 3$ ) and corresponding to a random matrix representation of the tensor-valued random field 
$\mathbf{x} \mapsto[[\mathbf{L}(\mathbf{x})]]$

Let $\mathbf{x} \mapsto[\underline{L}(\mathbf{x})]$ be the mean value of random field $\mathbf{x} \mapsto[\mathbf{L}(\mathbf{x})]$, defined from $\Omega$ into $\mathbb{M}_{n}^{+}(\mathbb{R})$ and supposed to be known. Eigenvalues are assumed to be sorted in increasing order throughout this paper, making use of order statistics in the random case. For $\mathbf{x}$ fixed in $\Omega$, let $\left\{\underline{\lambda}_{i}(\mathbf{x})\right\}_{i=1}^{n}$ and $\left\{\underline{\varphi}^{i}(\mathbf{x})\right\}_{i=1}^{n}$ be the sets of associated eigenvalues and eigenvectors of $[\underline{L}(\mathbf{x})]$. Let $\mathcal{I}=\left\{\mathfrak{i}_{1}, \ldots, \mathfrak{i}_{m}\right\}$ be an index set of positive integers, such that $m \leq n$ and $1 \leq \mathfrak{i}_{k} \leq n$ for all $k$ in $[1, m]$. It is assumed that the variances of $m$ selected stochastic eigenvalues of random matrix $[\mathbf{L}(\mathbf{x})]$ are specified through the following constraint [13]:

$$
\mathrm{E}\left\{\left(\underline{\varphi}^{\mathfrak{i}_{k}}(\mathbf{x})^{\mathrm{T}}[\mathbf{L}(\mathbf{x})] \underline{\varphi}^{\mathfrak{i}_{k}}(\mathbf{x})\right)^{2}\right\}=s_{k}(\mathbf{x})^{2} \underline{\lambda}_{\mathfrak{i}_{k}}(\mathbf{x})^{2}
$$

for $k=1, \ldots, m$. The deterministic field $\mathbf{x} \mapsto s_{k}(\mathbf{x})$ is assumed to be known and can be either computed when full-field (displacement) measurements are available or supposed a priori when the experimental identification is carried out solving a boundary value problem.

Let $[\underline{\Lambda}(\mathbf{x})]$ be the $(m \times m)$ diagonal matrix the components of which are the eigenvalues of mean matrix $[\underline{L}(\mathbf{x})]$ corresponding to the constrained eigenvalues and let $[\underline{\Phi}(\mathbf{x})]$ be the $(n \times m)$ matrix whose columns are the corresponding eigenvectors:

$$
\begin{gathered}
{[\underline{\Lambda}(\mathbf{x})]=\left[\begin{array}{cccc}
\underline{\lambda}_{\mathbf{i}_{1}}(\mathbf{x}) & 0 & \cdots & 0 \\
0 & \underline{\lambda}_{\mathfrak{i}_{2}}(\mathbf{x}) & \ddots & \vdots \\
\vdots & \ddots & \ddots & 0 \\
0 & \ldots & 0 & \underline{\lambda}_{i_{m}}(\mathbf{x})
\end{array}\right],} \\
{[\underline{\Phi}(\mathbf{x})]=\left[\underline{\varphi}^{\mathfrak{i}_{1}}(\mathbf{x}) \ldots \underline{\varphi}^{\mathfrak{i}_{m}}(\mathbf{x})\right] .}
\end{gathered}
$$

Let $[\underline{N}(\mathbf{x})]$ be the $(n \times n)$ matrix defined as follows:

- If $m<n,[\underline{N}(\mathbf{x})]$ is the following block matrix:

$$
[\underline{N}(\mathbf{x})]=\left[\left[\underline{N}_{1}(\mathbf{x})\right] \quad\left[\underline{N}_{2}(\mathbf{x})\right]\right],
$$

in which the deterministic matrices $\left[\underline{N}_{1}(\mathbf{x})\right]$ and $\left[\underline{N}_{2}(\mathbf{x})\right]$ are defined as:

$$
\left[\underline{N}_{1}(\mathbf{x})\right]=[\underline{L}(\mathbf{x})][\underline{\Phi}(\mathbf{x})][\underline{\Lambda}(\mathbf{x})]^{-1 / 2}, \quad\left[\underline{N}_{2}(\mathbf{x})\right]=[\underline{D}(\mathbf{x})],
$$

and where the $(n \times(n-m))$ matrix $[\underline{D}(\mathbf{x})]$ is such that:

$$
[\underline{D}(\mathbf{x})][\underline{D}(\mathbf{x})]^{\mathrm{T}}=[\underline{L}(\mathbf{x})]-[\underline{L}(\mathbf{x})][\underline{\Phi}(\mathbf{x})][\underline{\Lambda}(\mathbf{x})]^{-1}[\underline{\Phi}(\mathbf{x})]^{\mathrm{T}}[\underline{L}(\mathbf{x})] .
$$

- If $m=n$ (that is to say, if all the eigenvalues are constrained), $[\underline{N}(\mathbf{x})]$ is defined as:

$$
[\underline{N}(\mathbf{x})]=[\underline{L}(\mathbf{x})][\underline{\Phi}(\mathbf{x})][\underline{\Lambda}(\mathbf{x})]^{-1 / 2} .
$$

For $\mathbf{x} \in \Omega$, the random matrix $[\mathbf{L}(\mathbf{x})]$ is defined as:

$$
[\mathbf{L}(\mathbf{x})]=[\underline{N}(\mathbf{x})][\mathbf{G}(\mathbf{x})][\underline{N}(\mathbf{x})]^{\mathrm{T}},
$$


in which $\mathbf{x} \mapsto[\mathbf{G}(\mathbf{x})]$ is a random field defined on probability space $(\Theta, \mathcal{T}, P)$, indexed by $\Omega$ and whose probabilistic model can be readily deduced from the one associated with $\mathbf{x} \mapsto[\mathbf{L}(\mathbf{x})]$. For all $\mathbf{x}$ in $\Omega$, the random matrix $[\mathbf{G}(\mathbf{x})]$ is finally written as:

$$
[\mathbf{G}(\mathbf{x})]=[\mathbf{H}(\mathbf{x})][\mathbf{H}(\mathbf{x})]^{\mathrm{T}},
$$

where the underlying random field $\mathbf{x} \mapsto[\mathbf{H}(\mathbf{x})]$, defined on probability space $(\Theta, \mathcal{T}, P)$ and indexed by $\Omega$, is defined, following the overall methodology introduced in [24] [25], through a nonlinear mapping of a set of $n(n+1) / 2$ independent Gaussian stochastic germs. From a methodological point of view, the latter will be used to induce a correlation structure on non-Gaussian random field $\mathbf{x} \mapsto[\mathbf{L}(\mathbf{x})]$ (see Section 2.2) and for $\mathbf{x}$ fixed in $\Omega$, subsequently mapped onto selected components of random matrix $[\mathbf{H}(\mathbf{x})]$ (see Section $2.3)$.

\subsection{Definition of a family of stochastic germs $\left\{\mathbf{x} \mapsto U_{i \ell}(\mathbf{x})\right\}_{i, \ell}$}

Let $\left\{\mathbf{x} \mapsto U_{i \ell}(\mathbf{x})\right\}_{1 \leq \ell \leq i \leq n}$ be a set of $n(n+1) / 2$ independent second-order centered homogeneous Gaussian random fields, defined on probability space $(\Theta, \mathcal{T}, P)$, indexed by $\mathbb{R}^{d}$ and with values in $\mathbb{R}$, such that for all $\mathbf{x}$ in $\mathbb{R}^{d}, \mathrm{E}\left\{U_{i \ell}(\mathbf{x})^{2}\right\}=1$. This family of stochastic germs will be used to define the random field $\mathbf{x} \mapsto[\mathbf{H}(\mathbf{x})]$, as explained in Section 2.3. For $\mathbf{y} \in \mathbb{R}^{d}$, let $\left\{\mathbf{y} \mapsto \mathrm{R}_{U_{i \ell}}(\mathbf{y})\right\}$ be the set of associated autocorrelation functions, defined by:

$$
\mathrm{R}_{U_{i \ell}}(\mathbf{y})=\mathrm{E}\left\{U_{i \ell}(\mathbf{x}+\mathbf{y}) U_{i \ell}(\mathbf{x})\right\}, \quad \mathrm{R}_{U_{i \ell}}(0)=1 .
$$

In order to reduce the parametrization of the model, each autocorrelation function $\mathbf{y} \mapsto \mathrm{R}_{U_{i \ell}}(\mathbf{y})$ is written as:

$$
\mathrm{R}_{U_{i \ell}}(\mathbf{y})=\prod_{k=1}^{d} r_{k}^{i \ell}\left(y_{k}\right)
$$

where $r_{k}^{i \ell}(0)=1$ and $r_{k}^{i \ell}\left(y_{k}\right)=\left(2 L_{k}^{i \ell} /\left(\pi y_{k}\right)\right)^{2} \sin ^{2}\left(\pi y_{k} /\left(2 L_{k}^{i \ell}\right)\right)$ for $y_{k} \neq 0$. As an illustration, the plot of function $\mathbf{y}=\left(y_{1}, y_{2}\right) \mapsto \mathrm{R}_{U_{i \ell}}(\mathbf{y})$ is shown on Fig. 1 for $L_{1}^{i \ell}=L_{2}^{i \ell}=0.33$.

Each stochastic germ $\mathbf{x} \mapsto U_{i \ell}(\mathbf{x})$ is then entirely defined by the set of $d$ spatial correlation lengths $\left\{L_{k}^{i \ell}\right\}_{k=1}^{d}$, defined as:

$$
L_{k}^{i \ell}=\int_{0}^{+\infty}\left|\mathrm{R}_{U_{i \ell}}\left(0, \ldots, y_{k}, \ldots, 0\right)\right| d y_{k} .
$$

\subsection{Definition of random field $\mathrm{x} \mapsto[\mathrm{H}(\mathrm{x})]$ : probability distributions}

Let $m<n$ and let $\mathbf{x}$ be fixed in $\mathbb{R}^{d}$. The lower triangular matrix $[\mathbf{H}(\mathbf{x})]$ is then a random variable with components $[\mathbf{H}]_{i \ell}$ (for the sake of clarity, the dependence on $\mathbf{x}$ is dropped out hereafter). Let $i$ be fixed in $\{1, \ldots, n\}$.

For $i>m$, the random variables $[\mathbf{H}]_{i 1}, \ldots,[\mathbf{H}]_{i i}$ are all independent from each other (and from the other elements). Consequently, their joint probability density function, defined from $\mathbb{R} \times \ldots \times \mathbb{R} \times \mathbb{R}^{+}$into $\mathbb{R}^{+}$, can be written as:

$$
p_{[\mathbf{H}]_{i 1}, \ldots,[\mathbf{H}]_{i i}}\left([H]_{i 1}, \ldots,[H]_{i i}\right)=\prod_{\ell=1}^{i} p_{[\mathbf{H}]_{i \ell}}\left([H]_{i \ell}\right),
$$




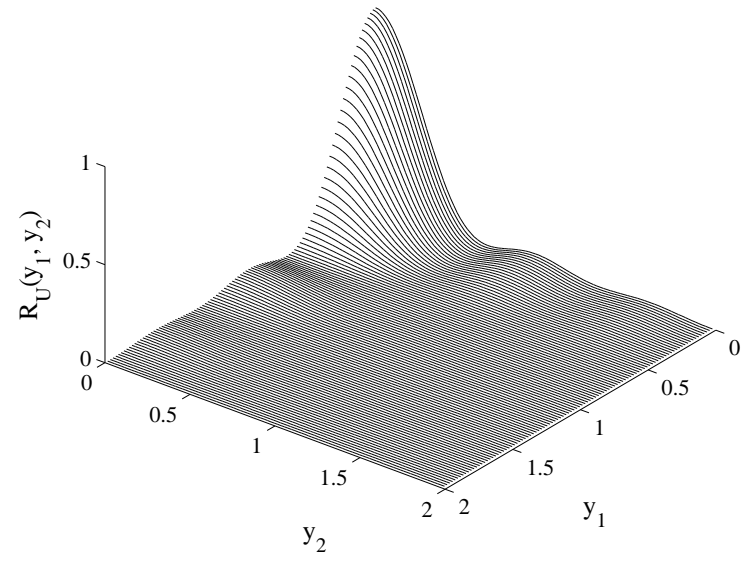

Figure 1: Plot of $\mathbf{y}=\left(y_{1}, y_{2}\right) \mapsto \mathrm{R}_{U_{i \ell}}(\mathbf{y})$ for $L_{1}^{i \ell}=L_{2}^{i \ell}=0.33$ and $\Omega=(] 0,2[)^{2}$.

where the random variables $[\mathbf{H}]_{i \ell}, i>\ell$, are normally distributed with mean 0 and standard deviation $1 / \sqrt{2 \mu_{i}(\mathbf{x})}$ and the random variable $[\mathbf{H}]_{i i}$ is distributed according to:

$$
p_{[\mathbf{H}]_{i i}}\left([H]_{i i}\right)=c_{1}[H]_{i i}{ }^{n-1-i+2 \alpha(\mathbf{x})} \exp \left[-\mu_{i}(\mathbf{x})[H]_{i i}{ }^{2}\right],
$$

in which:

- The real parameter $\alpha(\mathbf{x})$ is strictly positive.

- The parameter $\mu_{i}(\mathbf{x})$ is given by $\mu_{i}(\mathbf{x})=(n-1+2 \alpha(\mathbf{x})) / 2$.

- The real number $c_{1}=2 \mu_{i}(\mathbf{x})^{(n-i) / 2+\alpha(\mathbf{x})} / \Gamma((n-i) / 2+\alpha(\mathbf{x}))$ is the normalization constant and $t \mapsto \Gamma(t)$ is the Gamma function:

$$
\Gamma(t)=\int_{0}^{+\infty} z^{t-1} e^{-z} d z .
$$

For $i \leq m$, the random variables $\left\{[\mathbf{H}]_{i \ell}\right\}_{\ell=1}^{i}$ are statistically dependent. It should be pointed out however that the families of random variables $\left\{[\mathbf{H}]_{11}\right\},\left\{[\mathbf{H}]_{21},[\mathbf{H}]_{22}\right\}, \ldots,\left\{[\mathbf{H}]_{m \ell}, \ell=1, \ldots, m\right\}$ are independent. Their joint probability density function is defined as:

$$
\begin{aligned}
p_{[\mathbf{H}]_{i 1}, \ldots,[\mathbf{H}]_{i i}}\left([H]_{i 1}, \ldots,[H]_{i i}\right)= & c_{2}[H]_{i i}{ }^{n-1-i+2 \alpha(\mathbf{x})} \\
& \times \exp \left(-\mu_{i}(\mathbf{x})\left(\sum_{\ell=1}{ }^{i}[H]_{i \ell}{ }^{2}\right)\right. \\
& \left.-\tau_{i}(\mathbf{x})\left(\sum_{\ell=1}^{i}[H]_{i \ell}{ }^{2}\right)^{2}\right),
\end{aligned}
$$


where $c_{2}$ is a normalization constant. The parameter $\mu_{i}(\mathbf{x}), i \leq m$, can further be written as a function of parameters $\alpha(\mathbf{x})$ and $\tau_{i}(\mathbf{x})$ by enforcing the following equation to hold:

$$
\int_{\mathbb{R}_{*}^{+}} c_{3} g^{(n+2 \alpha(\mathbf{x})-1) / 2} \exp \left\{-\mu_{i}(\mathbf{x}) g-\tau_{i}(\mathbf{x}) g^{2}\right\} d g=1,
$$

where

$$
c_{3}=1 / \int_{\mathbb{R}_{*}^{+}} g^{(n+2 \alpha(\mathbf{x})-3) / 2} \exp \left\{-\mu_{i}(\mathbf{x}) g-\tau_{i}(\mathbf{x}) g^{2}\right\} d g .
$$

Let $\mathbf{H}^{(i)}$ and $\mathbf{U}^{(i)}$ be the random vectors with components $\left(H_{i 1}, \ldots, H_{i i}\right)$ and $\left(U_{i 1}, \ldots, U_{i i}\right)$, respectively. The random vector $\mathbf{U}^{(i)}$ is such that:

$$
\mathrm{E}\left\{\mathbf{U}^{(i)}\right\}=\mathbf{0}, \quad \mathrm{E}\left\{\mathbf{U}^{(i)} \mathbf{U}^{(i)^{\mathrm{T}}}\right\}=\left[I_{i}\right]
$$

with $\left[I_{i}\right]$ the $i \times i$ identity matrix. We now introduce the nonlinear mapping $f^{(i)}$ from $\mathbb{R}^{i}$ into $\mathcal{C}_{\mathbf{H}^{(i)}}=$ $\mathbb{R} \times \ldots \times \mathbb{R} \times \mathbb{R}^{+}$, such that $\mathbf{H}^{(i)}=f^{(i)}\left(\mathbf{U}^{(i)}\right)$.

\subsection{Definition of the nonlinear mapping for $i>m$}

For $i>m$, the mapping can be readily defined on each component:

- For $\ell=1, \ldots, i-1$, one has:

$$
[\mathbf{H}]_{i \ell}=\frac{U_{i \ell}}{\sqrt{2 \mu_{i}(\mathbf{x})}}
$$

- For $\ell=i$, one has:

$$
[\mathbf{H}]_{i i}=\sqrt{\frac{F_{\mathcal{G}((n-i+2 \alpha(x)) / 2,1)}^{-1}\left(F_{\mathcal{N}(0,1)}\left(U_{i i}\right)\right)}{\mu_{i}(\mathbf{x})}},
$$

where $t \mapsto F_{\mathcal{G}(a, b)}^{-1}(t)$ and $t \mapsto F_{\mathcal{N}(0,1)}(t)$ are the Gamma inverse cumulative distribution function with parameters $(a, b) \in \mathbb{R}_{*}^{+2}$ and the standard normal cumulative distribution function, respectively.

In Eqs. $(20-21), \mu_{i}(\mathbf{x})$ is given by $\mu_{i}(\mathbf{x})=(n-1+2 \alpha(\mathbf{x})) / 2$.

\subsection{Definition of the nonlinear mapping for $i \leq m$}

For $i \leq m$, the random variables $\left\{[\mathbf{H}]_{i \ell}\right\}_{\ell=1}^{i}$ are jointly distributed w.r.t. to Eq. (16). Consequently, $f^{(i)}$ now defines a mapping of $i$ independent Gaussian random variables (as $\mathbf{x}$ is fixed) onto a $\mathcal{C}_{\mathbf{H}^{(i)}{ }^{-}}$ valued random variable and both the change of variable and simulation strategy (relying on a rejection algorithm) proposed in [13] (for random matrices) cannot be neither used nor extended (because of the spatial correlations of the Gaussian germs). It then follows that the definition of mapping $f^{(i)}, i \leq m$, requires a more specific methodology and two possible strategies are discussed below. 


\subsubsection{Use of a probabilistic transformation.}

A first approach consists in defining the mapping $f^{(i)}$ through the Rosenblatt transformation [19]:

$$
\begin{aligned}
{\left[\mathbf{H}_{i 1}\right] } & =P_{\left[\mathbf{H}_{i 1}\right]}^{-1}\left(F_{\mathcal{N}(0,1)}\left(U_{i 1}\right)\right), \\
{\left[\mathbf{H}_{i 2}\right] } & =P_{\left[\mathbf{H}_{i 2}\right] \mid\left[\mathbf{H}_{i 1}\right]}^{-1}\left(F_{\mathcal{N}(0,1)}\left(U_{i 2}\right)\right), \\
\ldots & , \\
{\left[\mathbf{H}_{i i}\right] } & =P_{\left[\mathbf{H}_{i i}\right] \mid\left[\mathbf{H}_{i(i-1)}\right], \ldots,\left[\mathbf{H}_{i 1}\right]}{ }^{-1}\left(F_{\mathcal{N}(0,1)}\left(U_{i i}\right)\right),
\end{aligned}
$$

where $\left[H_{i \ell}\right] \mapsto P_{\left[\mathbf{H}_{i \ell}\right] \mid,\left[\mathbf{H}_{i(\ell-1)}\right] \ldots,\left[\mathbf{H}_{i 1}\right]}\left(\left[H_{i \ell}\right]\right), \ell=2, \ldots, i$, is the conditional probability distribution function

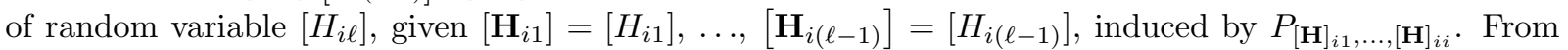
Eq. (22), it is seen that the probabilistic transformation must be carried out sequentially, solving first for $\left[\mathbf{H}_{i 1}\right]$, then for $\left[\mathbf{H}_{i 2}\right]$ given $\left[\mathbf{H}_{i 1}\right]$, etc.

It is worth noticing that (i) the use of the probabilistic model in either forward simulations or inverse problem solving (which may rely on an iterative optimization algorithm) requires performing multidimensional integrations with some potential numerical bias due to the particular form of the joint probability density function (with an obvious dependence on the values of parameters $\alpha(\mathbf{x})$ and $\mu_{i}(\mathbf{x})$; see Eq. (16)) and that (ii) such a transformation has to be performed $m$ times, at each point $\mathbf{x}$ of the domain under consideration and for all the realizations of the stochastic germs. These facts can clearly yield a prohibitive computational cost and make such an approach be restricted to small values of $m$ (that is to say, when only a few eigenvalues are constrained) and to a reasonable number of numerical Monte Carlo simulations. Consequently, we propose below another strategy which turns out to be more computational effective.

\subsubsection{Use of a statistical reduction and projection onto the polynomial chaos.}

We assume that $N_{\text {data }}$ realizations of random vector $\mathbf{H}^{(i)}$ can be synthesized, using either an inverse probabilistic transform, a rejection method or a Markov Chain Monte Carlo sampling for instance. Let $\underline{\mathbf{h}}^{(i)}=\mathrm{E}\left\{\mathbf{H}^{(i)}\right\}$ and $\left[C_{\mathbf{H}^{(i)}}\right]=\mathrm{E}\left\{\left(\mathbf{H}^{(i)}-\underline{\mathbf{h}}^{(i)}\right)\left(\mathbf{H}^{(i)}-\underline{\mathbf{h}}^{(i)}\right)^{\mathrm{T}}\right\}$ be the mean value and covariance matrix of $\mathbf{H}^{(i)}$, estimated from the above realizations. Note that parameter $N_{\text {data }}$ has to be determined from a convergence analysis on these moment estimates, so that no additional bias is introduced through the statistical reduction. Let $[V]=\left[\mathbf{v}^{1}, \ldots, \mathbf{v}^{N_{K L}}\right]$ be the $\left(i \times N_{K L}\right)$ real matrix constituted by the $N_{K L}$ eigenvectors of $\left[C_{\mathbf{H}^{(i)}}\right]$ corresponding to the $N_{K L}$ largest associated eigenvalues $\lambda_{1} \geq \ldots \geq \lambda_{N_{K L}}$, such that $[V]^{\mathrm{T}}[V]=\left[I_{i}\right]$. The truncated Karhunen-Loève expansion (KLE) of random vector $\mathbf{H}^{(i)}$ then reads:

$$
\mathbf{H}^{(i)} \simeq \underline{\mathbf{h}}^{(i)}+\sum_{j=1}^{N_{K L}} \sqrt{\lambda_{j}} \eta_{j} \mathbf{v}^{j},
$$

where $\left\{\eta_{j}\right\}_{j=1}^{N_{K L}}$ is a set of uncorrelated (but generally statistically dependent) orthogonal centered random variables whose arbitrary joint probability distribution is estimated having recourse to a projection onto the polynomial chaos. Since the length of the Gaussian germ used for defining the mapping is $i$, it further follows that the resulting interpolated second-order vector-valued random variable $\boldsymbol{\eta}=\left(\eta_{1}, \ldots, \eta_{N_{K L}}\right)$ has to be of length $N_{K L} \geq i$. From $\max \left\{N_{K L}\right\}=i$, we then deduce $N_{K L}=i$. Consequently, $\boldsymbol{\eta}$ admits the following Gaussian polynomial chaos expansion, truncated at order $N_{\text {ord }}$ [31] (see also [7] [29]):

$$
\boldsymbol{\eta}^{p c e} \simeq \sum_{\gamma=1}^{N_{p c e}} \mathbf{z}^{\gamma} \Psi_{\gamma}\left(\mathbf{U}^{(i)}\right),
$$


where $N_{\text {pce }}=\left(i+N_{\text {ord }}\right) ! /\left(i ! N_{\text {ord }} !\right)-1$ and:

- $\left\{\mathbf{z}^{\gamma}\right\}_{\gamma=1}^{N_{p c e}}$ is a set of deterministic $\mathbb{R}^{i}$-valued coefficients such that,

$$
\sum_{\gamma=1}^{N_{p c e}} \mathbf{z}^{\gamma} \mathbf{z}^{\gamma \mathrm{T}}=\left[I_{i}\right]
$$

- The random variables $\left\{\Psi_{\gamma}\left(\mathbf{U}^{(i)}\right)\right\}_{\gamma=1}^{N_{p c e}}$ are the normalized Hermite polynomials (renumbered for notational convenience) of the $\mathbb{R}^{i}$-valued Gaussian random variable $\mathbf{U}^{(i)}$ such that,

$$
\mathrm{E}\left\{\Psi_{\gamma}\left(\mathbf{U}^{(i)}\right)\right\}=0, \quad \mathrm{E}\left\{\Psi_{\gamma}\left(\mathbf{U}^{(i)}\right) \Psi_{\beta}\left(\mathbf{U}^{(i)}\right)\right\}=\delta_{\gamma \beta},
$$

with $\delta_{\gamma \beta}$ the kronecker symbol.

The Maximum Likelihood Method [21] is used to estimate the chaos coefficients (see [6] [5] for instance). In this context, note that the KLE is basically used here to make the random variables $\eta_{1}, \ldots$, $\eta_{N_{K L}}$ uncorrelated, in order to allow an identification of the chaos coefficients by using the Maximum Likelihood Method on first-order marginal probability density functions, as discussed in [6] and [28]. Let $\left\{\boldsymbol{\eta}^{\text {sim }}\left(\theta_{k}\right)\right\}_{1}^{N_{\text {data }}}$ be the set of independent realizations of random vector $\boldsymbol{\eta}$ computed from the ones of $\mathbf{H}^{(i)}$ by using the orthogonality of the eigenvectors of $\left[C_{\mathbf{H}^{(i)}}\right]$, for $j=1, \ldots, i$ and $k=1, \ldots, N_{d a t a}$ :

$$
\eta_{j}^{s i m}\left(\theta_{k}\right)=\frac{1}{\sqrt{\lambda_{j}}}<\mathbf{H}^{(i)}\left(\theta_{k}\right)-\underline{\mathbf{h}}^{(i)}, \mathbf{v}^{j}>_{\mathbb{R}^{i}}
$$

where $\langle\cdot, \cdot\rangle_{\mathbb{R}^{i}}$ denotes the Euclidean inner product in $\mathbb{R}^{i}$. Taking advantage of the fact that random variables $\eta_{1}, \ldots, \eta_{i}$ are uncorrelated, let $\left(\mathbf{z}^{1}, \ldots, \mathbf{z}^{N_{p c e}}\right) \mapsto \mathcal{L}\left(\mathbf{z}^{1}, \ldots, \mathbf{z}^{N_{p c e}}\right)$ be the following log-likelihood function,

$$
\mathcal{L}\left(\mathbf{z}^{1}, \ldots, \mathbf{z}^{N_{\text {pce }}}\right)=\sum_{j=1}^{i} \sum_{k=1}^{N_{\text {data }}} \log _{10}\left(p_{\eta_{j}^{p c e}}\left(\eta_{j}^{\text {sim }}\left(\theta_{k}\right) ; \mathbf{z}^{1}, \ldots, \mathbf{z}^{N_{p c e}}\right)\right),
$$

in which the marginal probability density function $\eta_{j} \mapsto p_{\eta_{j}^{p c e}}\left(\eta_{j}\right)$ can be estimated from numerical Monte Carlo simulations using the kernel estimation method for instance. The Maximum Likelihood estimate $\left(\widehat{\mathbf{z}}^{1}, \ldots, \widehat{\mathbf{z}}^{N_{p c e}}\right)$ of the deterministic coefficients is then defined as:

$$
\left(\widehat{\mathbf{z}}^{1}, \ldots, \widehat{\mathbf{z}}^{N_{p c e}}\right)=\underset{\mathcal{S}}{\arg \max } \mathcal{L}\left(\mathbf{z}^{1}, \ldots, \mathbf{z}^{N_{p c e}}\right)
$$

where $\mathcal{S}$ denotes the admissible set satisfying the constraint defined by Eq. (25). The non-convex optimization problem (29) has to be solved by a random search strategy. In this paper, we use the numerical strategy proposed in [27], where recurrent optimization problems on first-order marginal density functions are sequentially solved using a Latin-hypercube-sampling-like algorithm. Taking into account that $\mathrm{E}\left\{\boldsymbol{\eta}^{p c e}\right\}=\mathbf{0}, \mathrm{E}\left\{\boldsymbol{\eta}^{p c e} \boldsymbol{\eta}^{p c e \mathrm{~T}}\right\}=\left[I_{i}\right]$ and invoking the Maximum Entropy Principle, the random search is performed in the neighborhood of a Gaussian prior distribution whose mean vector and covariance matrix are the null vector $\mathbf{0}$ and the $(i \times i)$ identity matrix, respectively. Note finally that in the present case, the convergence analysis of the chaos representation has to be carried out with respect to the order of 
expansion only, since the length of the Gaussian germ $\mathbf{U}^{(i)}$ is fixed. Such an analysis can be performed by introducing the error function $N_{\text {ord }} \mapsto \epsilon_{j}^{p c e}\left(N_{\text {ord }}\right)$ on coordinate $\eta_{j}$ defined as [27]:

$$
\epsilon_{j}^{\text {pce }}\left(N_{\text {ord }}\right)=\int_{s p_{j}}\left|\log _{10}\left\{p_{\eta_{j}^{\text {sim }}}(e)\right\}-\log _{10}\left\{p_{\eta_{j}^{p c e}}\left(e ; \mathbf{z}^{1}, \ldots, \mathbf{z}^{N_{\text {pce }}}\right)\right\}\right| d e,
$$

in which $s p_{j}$ is the support defined as the intersection of the supports of $e \mapsto p_{\eta_{j}^{s i m}}(e)$ and $e \mapsto$ $p_{\eta_{j}^{p c e}}\left(e ; \mathbf{z}^{1}, \ldots, \mathbf{z}^{N_{p c e}}\right)$. The overall convergence of the chaos expansion can also be studied by characterizing the convergence of the global error function $N_{\text {ord }} \mapsto \operatorname{Conv}\left(N_{\text {ord }}\right)$ given by:

$$
\operatorname{Conv}\left(N_{\text {ord }}\right)=\frac{1}{i} \sum_{j=1}^{i} \epsilon_{j}^{p c e}\left(N_{\text {ord }}\right) .
$$

An appropriate order of expansion can thus be selected by choosing a maximal value, either for all the parameters $\epsilon_{j}^{p c e}$ (say, 0.5 for instance) or for parameter $\operatorname{Conv}\left(N_{\text {ord }}\right)$. Once the coefficients of the projection have been identified, vectorial representations of Eqs. (23 - 24) allow realizations of random vector $\mathbf{H}^{(i)}$ to be generated very efficiently at any point $\mathbf{x}$ of the domain and at a very low computational cost, since realizations of the Hermite polynomials can be computed and stored beforehand.

\subsection{Fundamental properties of random field $\mathrm{x} \mapsto[\mathrm{G}(\mathrm{x})]$}

The following properties of random field $\mathbf{x} \mapsto[\mathbf{G}(\mathbf{x})]$ can be deduced from the previous sections and from [13].

Property 2.6.1 $\forall \mathbf{x} \in \mathbb{R}^{d},[\mathbf{G}(\mathbf{x})] \in \mathbb{M}_{n}^{+}(\mathbb{R})$ almost surely.

Property 2.6.2 $\forall \mathbf{x} \in \mathbb{R}^{d}, \mathrm{E}\left\{\|[\mathbf{G}(\mathbf{x})]\|_{\mathrm{F}}^{2}\right\}<+\infty$, that is to say, $\mathbf{x} \mapsto[\mathbf{G}(\mathbf{x})]$ is a second-order random field.

Property 2.6.3 $\mathrm{x} \mapsto[\mathbf{G}(\mathbf{x})]$ is homogeneous for the translation in $\mathbb{R}^{d}$.

Property 2.6.4 The mean function $\mathbf{x} \mapsto \mathrm{E}\{[\mathbf{G}(\mathbf{x})]\}$ is independent of $\mathbf{x}$ and is such that $\forall \mathbf{x} \in \mathbb{R}^{d}$, $\mathrm{E}\{[\mathbf{G}(\mathbf{x})]\}=\left[I_{n}\right]$.

Property 2.6.5 The dispersion parameter $\delta_{[\mathbf{G}]}(\mathbf{x})$ defined as:

$$
\delta_{[\mathbf{G}]}(\mathbf{x})=\left(\frac{1}{n} \mathrm{E}\left\{\left\|[\mathbf{G}(\mathbf{x})]-\left[I_{n}\right]\right\|_{\mathrm{F}}^{2}\right\}\right)^{1 / 2},
$$

is such that:

$$
\delta_{[\mathbf{G}]}(\mathbf{x})^{2}=\frac{1}{n} \sum_{i=1}^{m} s_{i}(\mathbf{x})^{2}+\frac{n+1-(m / n)(n+1+2 \alpha(\mathbf{x}))}{n-1+2 \alpha(\mathbf{x})} .
$$

Taking into account Property 2.6.4, Eq. (32) implies that $\mathrm{E}\left\{\|[\mathbf{G}(\mathbf{x})]\|_{\mathrm{F}}^{2}\right\}=n\left(\delta_{[\mathbf{G}]}(\mathbf{x})+1\right)$. It can further be shown that $\forall i \leq m, s_{i}(\mathbf{x}) \rightarrow 1$ when $\alpha(\mathbf{x}) \rightarrow+\infty$. From Eq. (33), we deduce that $\delta_{[\mathbf{G}]}(\mathbf{x}) \rightarrow 0$ and then, that $\mathrm{E}\left\{\|[\mathbf{G}(\mathbf{x})]\|_{\mathrm{F}}^{2}\right\} \rightarrow n$ when $\alpha(\mathbf{x}) \rightarrow+\infty$. 
Property 2.6.6 Let $[\mu(\mathbf{x})]$ be the diagonal matrix with components $\mu_{i}(\mathbf{x})$. For $\mathbf{x}$ fixed in $\mathbb{R}^{d}$, let $[G] \mapsto$ $p_{[\mathbf{G}(\mathbf{x})]}([G])$ be the probability density function from $\mathbb{M}_{n}^{+}(\mathbb{R})$ into $\mathbb{R}^{+}$defining the probability distribution $P_{[\mathbf{G}(\mathbf{x})]}=p_{[\mathbf{G}(\mathbf{x})]}([G]) \mathrm{d} G$ of random matrix $[\mathbf{G}(\mathbf{x})]$, where the volume measure $\mathrm{d} G$ on $\mathbb{M}_{n}^{S}(\mathbb{R})$ is written as $\mathrm{d} G=2^{n(n-1) / 4} \prod_{1<i \leq \ell \leq n} \mathrm{~d}[G]_{i \ell}\left(\mathrm{d}[G]_{i \ell}\right.$ is the Lebesgue measure on $\mathbb{R}$; see [23]). The probability density function $p_{[\mathbf{G}(\mathbf{x})]}([G])$ is then given by:

$$
\begin{aligned}
p_{[\mathbf{G}(\mathbf{x})]}([G])= & c_{4}(\operatorname{det}([G]))^{\alpha(\mathbf{x})-1} \exp \left(-\operatorname{tr}\left([\mu(\mathbf{x})]^{\mathrm{T}}[G]\right)\right. \\
& \left.-\sum_{i=1}^{m} \tau_{i}(\mathbf{x})[G]_{i i}{ }^{2}\right),
\end{aligned}
$$

in which $c_{4}$ is a normalization constant.

Property 2.6.7 Random field $\mathbf{x} \mapsto[\mathbf{G}(\mathbf{x})]$ is mean-square continuous on $\mathbb{R}^{d}$.

Property 2.6.8 Realizations of random field $\mathbf{x} \mapsto[\mathbf{G}(\mathbf{x})]$ are continuous from $\mathbb{R}^{d}$ into $\mathbb{M}_{n}^{+}(\mathbb{R})$ almost surely.

Property 2.6.9 For all $(\boldsymbol{x}, \boldsymbol{\eta}) \in \mathbb{R}^{d} \times \mathbb{R}^{d}$, the tensor-valued covariance function $(\boldsymbol{x}+\boldsymbol{\eta}, \boldsymbol{x}) \mapsto \mathrm{C}_{[\boldsymbol{G}(\boldsymbol{x})]}(\boldsymbol{x}+$ $\boldsymbol{\eta}, \boldsymbol{x})$ is independent of $\boldsymbol{x}$ and is such that:

$$
\mathrm{C}_{[\boldsymbol{G}(\boldsymbol{x})]}(\boldsymbol{x}+\boldsymbol{\eta}, \boldsymbol{x})=\mathrm{C}_{[\boldsymbol{G}(\boldsymbol{x})]}^{*}(\boldsymbol{\eta})=\mathrm{E}\left\{\left([\boldsymbol{G}(\boldsymbol{x}+\boldsymbol{\eta})]-\left[I_{n}\right]\right) \otimes\left([\boldsymbol{G}(\boldsymbol{x})]-\left[I_{n}\right]\right)\right\},
$$

in which $\boldsymbol{\eta} \mapsto \mathrm{C}_{[\boldsymbol{G ( x ) ]}}^{*}(\boldsymbol{\eta})$ is a continuous function in $\mathbb{R}^{d}$.

\subsection{Fundamental properties of random field $\mathrm{x} \mapsto[\mathrm{L}(\mathrm{x})]$}

The following results can be deduced from the previous sections.

Property 2.7.1 $\forall \mathbf{x} \in \mathbb{R}^{d},[\mathbf{L}(\mathbf{x})] \in \mathbb{M}_{n}^{+}(\mathbb{R})$ almost surely.

Property 2.7.2 $\forall \mathbf{x} \in \mathbb{R}^{d}, \mathrm{E}\left\{\|[\mathbf{L}(\mathbf{x})]\|_{\mathrm{F}}^{2}\right\}<+\infty$, that is to say, $\mathbf{x} \mapsto[\mathbf{L}(\mathbf{x})]$ is a second-order random field.

From the definition of matrix $[\underline{N}(\mathbf{x})]$ (see Eqs. (4-7)) and using Property 2.6.4, one can deduce the following property.

Property 2.7.3 The mean function $\mathbf{x} \mapsto \mathrm{E}\{[\mathbf{L}(\mathbf{x})]\}$ is such that $\forall \mathbf{x} \in \mathbb{R}^{d}, \mathrm{E}\{[\mathbf{L}(\mathbf{x})]\}=[\underline{L}(\mathbf{x})]$.

From the construction above, it can be deduced that the random bilinear form associated with the weak formulation of the elasticity stochastic boundary value problem is non-uniformly elliptic [24]. However, it is interesting to note that because of the invertibility property, which implies the flatness of the p.d.f. of random matrix $[\mathbf{L}(\mathbf{x})]$ near lower bound $[0]$ (which is never reached), an uniform ellipticity condition can be recovered if one considers a new elasticity tensor random field $\mathbf{x} \mapsto[\widetilde{\mathbf{L}}(\mathbf{x})]=[\mathbf{L}(\mathbf{x})]+\varepsilon\left[I_{n}\right]$, in which $\varepsilon$ is a any positive constant independent of $\mathbf{x}$.

Finally, we introduce the field $\mathbf{x} \mapsto \delta_{[\mathbf{L}]}(\mathbf{x})$, allowing the level of statistical fluctuations of $\mathbf{x} \mapsto[\mathbf{L}(\mathbf{x})]$ to be characterized and defined as:

$$
\delta_{[\mathbf{L}]}(\mathbf{x})^{2}=\frac{\mathrm{E}\left\{\|[\mathbf{L}(\mathbf{x})]-[\underline{L}(\mathbf{x})]\|_{\mathrm{F}}^{2}\right\}}{\|[\underline{L}(\mathbf{x})]\|_{\mathrm{F}}^{2}} .
$$




\subsection{Influence of parameters $\alpha(\mathrm{x})$ and $\boldsymbol{\tau}(\mathrm{x})$}

The aim of this section is to characterize the influence of parameters $\mathbf{x} \mapsto \alpha(\mathbf{x})$ and $\mathbf{x} \mapsto \tau_{i}(\mathbf{x}), i \in \mathcal{I}$. For illustration purposes, let $\mathbf{x} \in \Omega$ be fixed, so that reference to spatial indexation is temporarily dropped out. Let $[\underline{L}]$ be the mean model defined as (unit is $[\mathrm{GPa}]$ ):

$$
[\underline{L}]=\left[\begin{array}{rrrrrr}
10.1036 & 0.5391 & 2.9625 & -0.0040 & 0.0071 & -0.0165 \\
& 10.1061 & 2.9782 & -0.0041 & -0.0070 & -0.0036 \\
& & 182.69 & 0.0197 & 0.0016 & 0.0145 \\
& & & 14.0339 & 0.0068 & 0.0008 \\
& \text { Sym. } & & & 14.0121 & -0.0103 \\
& & & & & 9.5552
\end{array}\right] .
$$

Eq. (37) corresponds to a random perturbation of the elasticity matrix of a carbon-epoxy unidirectional composite (with fibers aligned along axis $\mathbf{e}_{3}$ ).

First of all, numerical experiments, together with the results provided in Section 2.6, show that $\alpha \rightarrow$ $+\infty$ implies that $\delta_{[\mathbf{L}]} \rightarrow 0$ (that is to say, $[\mathbf{L}] \rightarrow[\underline{L}]$ in probability), while non-negligible levels of statistical fluctuations are obtained for $\alpha \leq 150$.

Let us now fix $\alpha$ to a given value, say, $\alpha=60$, and let $\boldsymbol{\tau}=(\tau, \tau, 0, \tau, \tau, 0)$. Consequently, the variances of stochastic eigenvalues $\lambda_{1}, \lambda_{2}, \lambda_{4}$ and $\lambda_{5}$ are similarly constrained, while no constraint is imposed on $\lambda_{3}$ and $\lambda_{6}$. The deterministic matrices $[\underline{\Phi}]$ and $[\underline{\Lambda}]$, introduced in Section 2.1, then read:

$$
\begin{gathered}
{[\underline{\Phi}]=\left[\begin{array}{llll}
\underline{\varphi}^{1} & \underline{\varphi}^{2} & \underline{\varphi^{4}} & \underline{\varphi}^{5}
\end{array}\right],} \\
{[\underline{\Lambda}]=\left[\begin{array}{llll}
\underline{\lambda}_{1} & 0 & 0 & 0 \\
0 & \underline{\lambda}_{2} & 0 & 0 \\
0 & 0 & \underline{\lambda}_{4} & 0 \\
0 & 0 & 0 & \underline{\lambda}_{5}
\end{array}\right] .}
\end{gathered}
$$

Let $\lambda \mapsto p_{\lambda_{i}}(\lambda)$ be the probability density function of random eigenvalue $\lambda_{i}$. The probability density functions of the stochastic eigenvalues are plotted for $\tau=1$ and $\tau=10^{4}$ in Fig. 2.

As expected, it is seen that the probability density functions of $\lambda_{3}$ and $\lambda_{6}$ are not affected by parameter $\tau$, although the coefficient of variation of $\lambda_{3}$ presents a very small variation which may be due to its closeness of the constrained eigenvalues. Furthermore, these figures show that for a given value of parameter $\alpha$ :

(i) Parameter $\tau_{i}$ has a negligible effect on the mean value of the stochastic eigenvalue $\lambda_{i}$.

(ii) Setting a large value of $\tau_{i}$ allows for a significant reduction of the variance of $\lambda_{i}$. At this stage, it is worth mentioning that no matter the value of $\tau_{i}$, the eigenvalue $\lambda_{i}$ still remains stochastic, so that its variance can be prescribed within a given range of values.

The coefficients of variation (estimated on a set of 2500 realizations by using mathematical statistics; see [21] for instance) of the six eigenvalues are plotted (in semi-log scale) on Fig. 3, parameter $\tau$ ranging from 0.1 to $10^{4}$.

It is seen that for $\tau \geq 10^{4}$, the coefficient of variation of $\lambda_{1}$ (resp. $\lambda_{2}, \lambda_{4}$ and $\lambda_{5}$ ) is reduced by approximately $25 \%$ (resp. $37 \%, 44 \%$ and $44 \%$ ). Beyond this value, all the coefficients of variation corresponding to constrained eigenvalues tend to a nonzero constant value, in agreement with the point (ii) mentioned above. The coefficients of variation of the unconstrained eigenvalues (namely $\lambda_{3}$ and $\lambda_{6}$ ) do not depend on $\tau$. Based on these comments, it can be finally concluded that: 

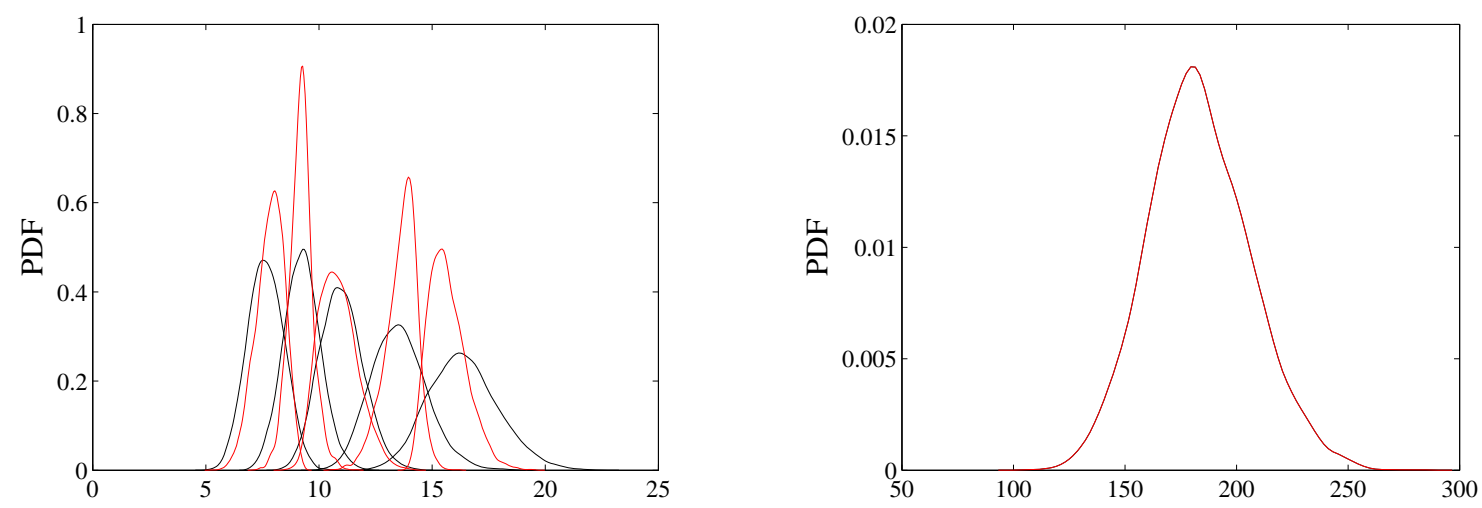

Figure 2: Plots of the probability density functions $\lambda \mapsto p_{\lambda_{i}}(\lambda)$ of the random eigenvalues $\lambda_{i}, i=1, \ldots, 5$ (left) and $\lambda_{6}$ (right), for $\alpha=60$. Black solid line: $\tau=1$. Red solid line: $\tau=10^{4}$.

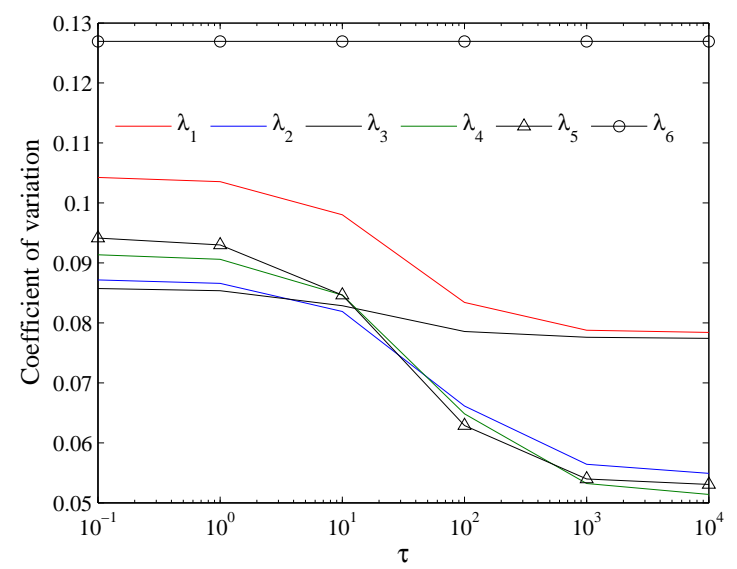

Figure 3: Plots of $\tau \mapsto \operatorname{CV}\left\{\lambda_{i}(\tau)\right\}$ for $\alpha=60$.

- The field $\mathbf{x} \mapsto \alpha(\mathbf{x})$ has to be used to calibrate the overall level of statistical fluctuations of random matrix $[\mathbf{L}(\mathbf{x})], \mathbf{x} \in \Omega$.

- The field $\mathbf{x} \mapsto \tau_{i}(\mathbf{x}), i \in \mathcal{I}$, allows the variance of random eigenvalue $\lambda_{i}$ to be partially prescribed. Furthermore, it is seen that the influence of $\tau_{i}(\mathbf{x})$ is significant over a very large range of values. 


\section{DEFINITION OF CONSTRAINTS WITH RESPECT TO MA- TERIAL SYMMETRY}

The aim of this section is to provide a mechanical insight in the way the variances of some eigenvalues may be constrained. For this purpose, we first define distances in the set of elasticity tensors, based on which a projection onto the class of elasticity tensors exhibiting given material symmetry can be defined. Such derivations turn out to be useful in order to quantify, in a probabilistic sense, the effect of variance constraints on material symmetry. Then, we illustrate the approach for the set of parameters defined in Section 2.8 .

\subsection{Distance and projection in the set of elasticity tensors}

The question of defining the distance between elasticity tensors has received considerable attention, especially within the context of experimental identification (see [3] for an application in geophysics for instance).

Several metrics have been introduced in the literature to quantify the distance between two elasticity tensors, the most widely used metrics being the Euclidean, Log-Euclidean [1] and Riemannian ones [14], denoted by $d_{E}, d_{L E}$ and $d_{R}$ respectively, and defined for any elasticity tensors [[ $\left.\left.L_{1}\right]\right]$ and $\left[\left[L_{2}\right]\right]$ by:

$$
\begin{gathered}
d_{E}\left(\left[\left[L_{1}\right]\right],\left[\left[L_{2}\right]\right]\right)=\left\|\left[\left[L_{2}\right]\right]-\left[\left[L_{1}\right]\right]\right\|, \\
d_{L E}\left(\left[\left[L_{1}\right]\right],\left[\left[L_{2}\right]\right]\right)=\left\|\log \left(\left[\left[L_{2}\right]\right]\right)-\log \left(\left[\left[L_{1}\right]\right]\right)\right\|, \\
d_{R}\left(\left[\left[L_{1}\right]\right],\left[\left[L_{2}\right]\right]\right)=\left\|\log \left(\left[\left[L_{1}\right]\right]^{-1 / 2}\left[\left[L_{2}\right]\right]\left[\left[L_{1}\right]\right]^{-1 / 2}\right)\right\|,
\end{gathered}
$$

in which the inner product and its associated norm are respectively given by:

$$
<<\left[\left[L_{1}\right]\right],\left[\left[L_{2}\right]\right]>>=\left[\left[L_{1}\right]\right]_{i j k \ell}\left[\left[L_{2}\right]\right]_{i j k \ell},
$$

and

$$
\|[[L]]\|=<<[[L]],[[L]]>>^{1 / 2} .
$$

Let $\mathcal{E}^{\mathrm{Sym}}$ be a class of elasticity tensors with given material symmetries (isotropy, transverse isotropy, orthotropy, etc.). Let $[[L]]$ be a fourth-order elasticity tensor having an arbitrary symmetry, with components $[[L]]_{i j k \ell}$ with respect to a given frame $\mathfrak{R}=\left(0, \mathbf{e}_{1}, \mathbf{e}_{2}, \mathbf{e}_{3}\right)$. We then denote by $\left[\left[L^{\mathrm{Sym}}\right]\right]=\mathcal{P}^{\mathrm{Sym}}([[L]])$ the projection of $[[L]]$ onto $\mathcal{E}^{\mathrm{Sym}}$, calculated by using one of the distance $d$ introduced above, such that:

$$
\left[\left[L^{\mathrm{Sym}}\right]\right]=\underset{[[\widetilde{L}]] \in \mathcal{E}^{\mathrm{Sym}}}{\arg \min } d([[L]],[[\widetilde{L}]]) .
$$

For an elasticity tensor with an arbitrary symmetry, solving Eq. (45) allows one to derive either closed-form expressions (when the Euclidean metric is used) for the closest tensors of higher symmetries, or equivalent optimization problems for the parameters of the projection spanning $\mathcal{E}^{\mathrm{Sym}}$ (when the Log-Euclidean or Riemannian distance is retained); see the discussion in [15]. In order to reduce at most the computation time, Euclidean projection will be used in the sequel (note however that once a projection scheme has been chosen, any of the metric introduced above can be used for characterizing the residual distance between a tensor with arbitrary symmetry and its projection, and that all the metrics qualitatively yield similar results). 


\subsection{Application}

The eigensystem coordinate-free characterization of the material symmetries [2] states that a material symmetry class can be defined by both the multiplicities of the eigenvalues and constraints on the related eigenspaces. Specifically, it can be shown for instance that an isotropic (resp. transversely isotropic) tensor has one eigenvalue of multiplicity five (resp. two eigenvalues of multiplicity two) and one eigenvalue of multiplicity one (resp. two eigenvalues of multiplicity one). In this context, it is interesting to note that the use of the classical random ensembles from the Random Matrix Theory generally implies all the stochastic eigenvalues to be of multiplicity one (because of the well-known repulsion phenomena) and that the corresponding random eigenspaces cannot be explicitly constrained nor described. Nevertheless, such a material symmetry characterization suggests that decreasing the variances of the random eigenvalues that would correspond to given deterministic eigenvalues of multiplicity higher than one, should the tensor exactly belong to the considered symmetry class, may allow some statistical properties of the distance to be prescribed. In fact, it can be shown that tailoring such variances to properly selected values allows the mean of the distance to be partially controlled, since one can then enforce, in some sense, the "relative closeness" of the relevant eigenvalues (see [8]).

In order to illustrate this fact, let $(\alpha, \boldsymbol{\tau}) \mapsto \mathrm{D}^{\mathrm{Sym}}(\alpha, \boldsymbol{\tau})$ be the function such that:

$$
\mathrm{D}^{\mathrm{Sym}}(\alpha, \boldsymbol{\tau})=\mathrm{E}\left\{d\left([\mathbf{L}],\left[\mathbf{L}^{\mathrm{Sym}}\right]\right)\right\},
$$

in which $[\mathbf{L}]$ (and implicitly, $\left[\mathbf{L}^{\mathrm{Sym}}\right]$ ) depends on $(\alpha, \boldsymbol{\tau})$ and $d$ is any of the distance previously introduced. We consider the set of parameters as defined in Section 2.8. Consequently, we consider the projection onto the set $\mathcal{E}^{\mathrm{TI}}$ of all the elasticity tensors exhibiting transverse isotropy with respect to $\mathbf{e}_{3}$, defined with respect to the Euclidean distance $d_{E}$ (see [15]). Following [2] and Section 2.8, the mean distance $\mathrm{D}^{\mathrm{TI}}$ can be specified, for a given overall level of statistical fluctuation, by setting an appropriate value of parameter $\tau$. The plot of function $\tau \mapsto \mathrm{D}^{\mathrm{TI}}(60,(\tau, \tau, 0, \tau, \tau, 0))$, obtained using the Riemannian distance, is shown in Fig. 4. It is seen that enforcing the closeness of the eigenvalues defining the considered material

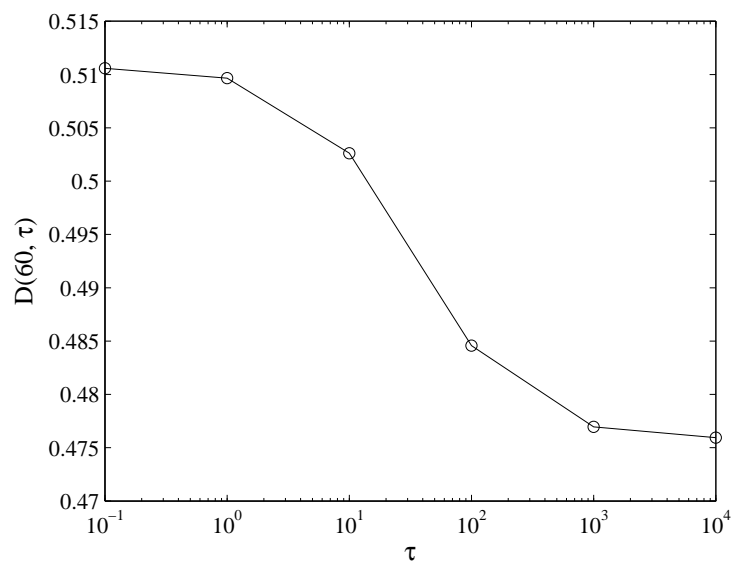

Figure 4: Plot of $\tau \mapsto \mathrm{D}^{\mathrm{TI}}(60,(\tau, \tau, 0, \tau, \tau, 0))$.

symmetry class (according to the coordinate-free characterization) allows for the reduction of the mean 
Riemannian distance. It can also be shown that this reduction is all the more important that the overall level of statistical fluctuation is high (that is to say, for small values of parameter $\alpha$ ). Unlike the few other probabilistic models previously derived within a nonparametric framework in the literature, the proposed probabilistic model thus allows to take into account, in some sense, higher statistical fluctuations in some given directions.

\section{APPLICATION}

\subsection{Numerical generation of an experimental database}

\subsubsection{Description of the stochastic boundary value problem.}

For this application, we consider the stochastic mesoscopic modeling of a random elastic microstructure, defined on mesoscale domain $\Omega=(] 0,1[)^{3}$ (with boundary $\left.\partial \Omega\right)$ of $\mathbb{R}^{3}(n=6)$. Consequently, random field $\mathbf{x} \mapsto[\mathbf{L}(\mathbf{x})]$ is now identified as the apparent elasticity tensor random field, constructed (and to be identified) at a given spatial resolution (that is to say, at a given mesoscale). For $\mathbf{x} \in \Omega$, the associated displacement second-order random field is denoted by $\mathbf{x}=\left(x_{1}, x_{2}, x_{3}\right) \mapsto \mathbf{V}(\mathbf{x})=\left(V_{1}(\mathbf{x}), V_{2}(\mathbf{x}), V_{3}(\mathbf{x})\right)$. For the given spatial resolution introduced above, we consider the finite element discretization of the associated elliptic stochastic boundary value problem (SBVP), for which the local random constitutive equation reads:

$$
\boldsymbol{\sigma}(\mathbf{x})=[\mathbf{L}(\mathbf{x})] \epsilon(\mathbf{x})
$$

in which $\boldsymbol{\sigma}(\mathbf{x})$ and $\boldsymbol{\epsilon}(\mathbf{x})$ (with $\left.\epsilon_{i \ell}(\mathbf{x})=\left(\partial V_{i}(\mathbf{x}) / \partial x_{\ell}+\partial V_{\ell}(\mathbf{x}) / \partial x_{i}\right) / 2\right)$ are the vectorial representations (defined to ensure the consistency with the matrix representation of the elasticity tensor) of the local random stress and strain tensors at point $\mathbf{x}$. We stress at this stage that the size of the finite element mesh used while spatially discretizing the model and the size of the spatial resolution associated with the given mesoscale are implicitly dependent (but not necessarily equal), since the choice of the mesoscale implies for instance some spatial correlation lengths (of the apparent elasticity tensor random field), for which four points are at least required (in each direction) to properly catch the correlation structure. As said previously, it is worth pointing out that the constructed prior probabilistic model to be used hereafter intrinsically depends on the choice of the mesoscale (as well as on the boundary conditions at microscale, the latter not being considered in the sequel since we are not concern with homogenization between microscale and mesoscale) but does not depend, neither on the geometry nor on the boundary conditions defined at this mesoscale. Consequently, since the model is used at the same, given mesoscale, the identification of its parameters can be performed solving the inverse stochastic problem related to the mesoscale boundary value problem (see below).

The prescribed Dirichlet boundary conditions are defined as follows:

- A null displacement condition is applied on $\partial \Omega_{0}$, where $\partial \Omega_{0}$ is constituted by the four points of coordinates $(0,0,0),(0,1,0),(1,0,0)$ and $(1,1,0)$.

- An arbitrary deterministic displacement condition $V(\mathbf{x})=V_{D}(\mathbf{x})$ is applied on $\partial \Omega_{D}$, where $\partial \Omega_{D}$ is the point of coordinates $(0,0,1)$.

The SBVP can be stated as follows: find the second-order random field $\mathbf{x} \mapsto \mathbf{V}(\mathbf{x})$, defined from $\Theta \times \bar{\Omega}$ into $\mathbb{R}^{3}$, such that:

$$
-\operatorname{div} \boldsymbol{\sigma}(\cdot)=0 \text { in } \Omega
$$




$$
\begin{gathered}
\mathbf{V}(\cdot)=0 \text { on } \partial \Omega_{0}, \\
\mathbf{V}(\cdot)=V_{D}(\cdot) \text { on } \partial \Omega_{D}, \\
\boldsymbol{\sigma}(\cdot) \boldsymbol{n}(\cdot)=\mathbf{0} \text { on } \partial \Omega_{N}=\partial \Omega \backslash \partial \Omega_{0} \cup \partial \Omega_{D} .
\end{gathered}
$$

in which $\boldsymbol{n}(\cdot)$ is the outward unit normal to $\partial \Omega_{N}$. The SBVP is solved by a non-intrusive stochastic finite elements method (that is to say, by coupling numerical Monte-Carlo simulations with a deterministic finite element solver). The domain $\Omega$ is meshed using 216 eight-nodes solid elements (with 8 integration points; see Fig. 5).

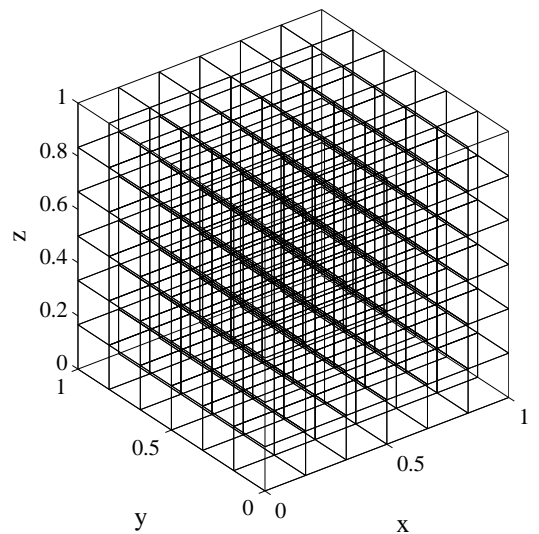

Figure 5: Finite Element mesh of domain $\Omega$.

For $N_{\text {exp }}$ realizations of random field $\mathbf{x} \mapsto[\mathbf{L}(\mathbf{x})]$ (see Section 4.1.2), the corresponding realizations of displacements $V_{2}$ and $V_{3}$ at the $N_{\text {nod }}$ nodes of the elements belonging to the face $\partial \Omega_{\text {obs }}=\left\{\mathbf{x} \in \Omega \mid x_{1}=0\right\}$ constitute the experimental database. For the sake of brevity, let $\mathbf{W}^{\exp }$ be the $\mathbb{R}^{2 N_{\text {nod }} \text {-valued random }}$ variable the components of which are the observable degrees of freedom. Consequently, the database is constituted by the set $\left\{\mathbf{w}_{i}^{\text {exp }}\right\}_{i=1}^{N_{e x p}}$ of experimental realizations of $\mathbf{W}^{\text {exp }}$. In order to be consistent with both usual experimental constraints and the inverse identification procedure, we set $N_{\text {exp }}=100$.

\subsubsection{Numerical Monte Carlo simulations of random field $\mathrm{x} \mapsto[\mathrm{L}(\mathrm{x})]$.}

The mean model is independent of $\mathbf{x}$ and is given by Eq. (37). We further assume that the spatial correlation lengths are such that $L_{1}^{i \ell}=L_{2}^{i \ell}=L_{3}^{i \ell}=L_{c}$ for $1 \leq \ell \leq i \leq n$. Finally, we consider the following constant fields of constraints:

$$
\begin{gathered}
\alpha(\mathbf{x})=\alpha=60, \\
\tau_{1}(\mathbf{x})=\tau_{2}(\mathbf{x})=\tau_{4}(\mathbf{x})=\tau_{5}(\mathbf{x})=200, \quad \tau_{3}(\mathbf{x})=\tau_{6}(\mathbf{x})=0,
\end{gathered}
$$

for all $\mathbf{x}$ in $\Omega$. The first step for generating realizations of random field $\mathbf{x} \mapsto[\mathbf{H}(\mathbf{x})]$ according to these parameters is the computation of the deterministic fields $\mathbf{x} \mapsto \mu_{i}(\mathbf{x}), i=1, \ldots, 6$. It follows from Eqs. (52-53) that $\mu_{i}(\mathbf{x})=\mu_{i}$ for all $\mathbf{x}$ in $\Omega$. For $i>4$, one has $\mu_{i}=(n-1+2 \alpha) / 2=62.5$ (see Section 2.3). For $i \leq 4$, the parameter $\mu_{i}$ can be computed by solving Eq. (17), making use of one of the two possible 
strategies briefly reviewed in [8] for instance. Alternatively, let $G_{i}$ be the random variable with values in $\mathbb{R}_{*}^{+}$whose probability density function $g \mapsto p_{G_{i}}(g)$, defined from $\mathbb{R}_{*}^{+}$into $\mathbb{R}$, is given by:

$$
p_{G_{i}}(g)=\widehat{\widehat{k}}_{i} g^{(n+2 \alpha-3) / 2} \exp \left\{-\mu_{i} g-\tau_{i} g^{2}\right\}
$$

where $\widehat{\widehat{k}}_{i}$ is the normalization constant. Eq. (17) can then be rewritten as:

$$
\mathrm{E}\left\{G_{i}\right\}=1 .
$$

As an example, Eq. (55) is solved combining a pattern search algorithm with Markov Chain Monte Carlo (MCMC) sampling (namely, the Metropolis-Hastings - MH- algorithm, with a thinned sequence and a burn-in period set to 2000). The proposal distribution is the normal probability density function centered at the mode of the distribution and whose coefficient of variation has been tuned beforehand. The convergence of the statistical estimate of the left-hand side (l.h.s.) of Eq. (55) with respect to the number of samples is illustrated on Fig. 6, for the best trial of the pattern search algorithm.

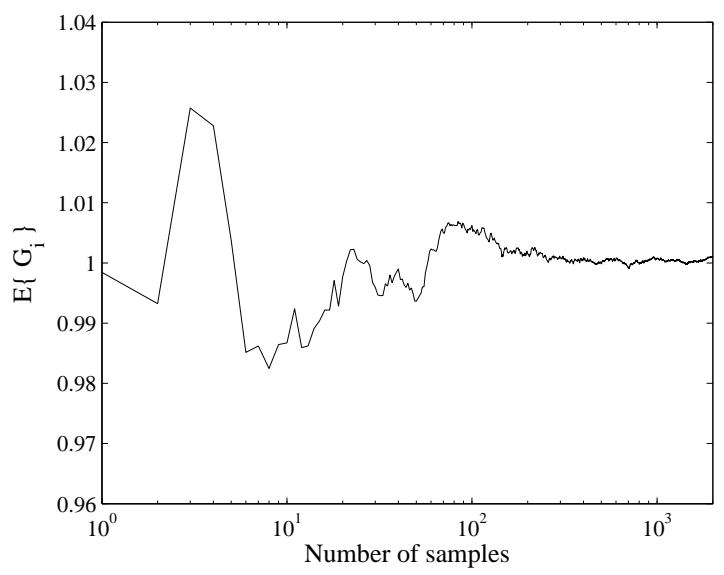

Figure 6: Convergence of the statistical estimate of $\mathrm{E}\left\{G_{i}\right\}$ with respect to the number of samples (Metropolis-Hastings algorithm).

Finally, one has $\mu_{i}=-337.9487$ for $i \leq 4$ (a symbolic calculation of the l.h.s. of Eq. (55) with $\alpha=60$, $\mu_{i}=-337.9487$ and $\tau_{i}=200$ yields 0.9991 ). It should be pointed out that the construction of the mapping $\left(\alpha, \tau_{i}\right) \mapsto \mu_{i}$ is independent of the problem under consideration (mean value, etc.) and thus, it may be carried out once for all (and in particular, out of the main optimization algorithm used for the inverse identification). The second step consists in performing a set of probabilistic transformations, mapping the independent realizations of the Gaussian germs $\left\{\mathbf{x} \mapsto U_{i \ell}(\mathbf{x})\right\}_{1 \leq \ell \leq i \leq n}$ into the corresponding independent realizations of random field $\mathbf{x} \mapsto[\mathbf{H}(\mathbf{x})]$ (see Section 2.4). For $\mathbf{x}$ fixed in $\Omega$, the nonlinear mappings for components $[\mathbf{H}]_{i \ell}$ and $[\mathbf{H}]_{i i}, i>m, \ell \leq i$, are computed using Eqs. (20) and (21). For $i \leq m$, we adopt the strategy relying on the projection onto the Gaussian polynomial chaos (see Eqs. (23) and (24)). This methodology requires the numerical Monte-Carlo simulations of random vector $\mathbf{H}^{(i)}$, in order to identify the coefficients involved in the chaos expansion (see Eq. (29)). While a few techniques for generating 
multivariate random variables are available in the literature, we recommend the use of a MCMC sampling technique. In particular, the slice sampling technique [16] turns out to be very efficient, since it does not require, unlike the $\mathrm{MH}$ algorithm, the definition of a proposal distribution (nor the definition of conditional distributions, used in Gibbs sampling for instance). In this study, we used the slice sampling technique with a burn-in period set to 500. Below, the overall methodology is exemplified for random vector $\mathbf{H}^{(4)}$. The convergence of the Euclidean norm of the mean vector estimate and that of the Frobenius norm of the covariance matrix estimate is first illustrated on Fig. 7.
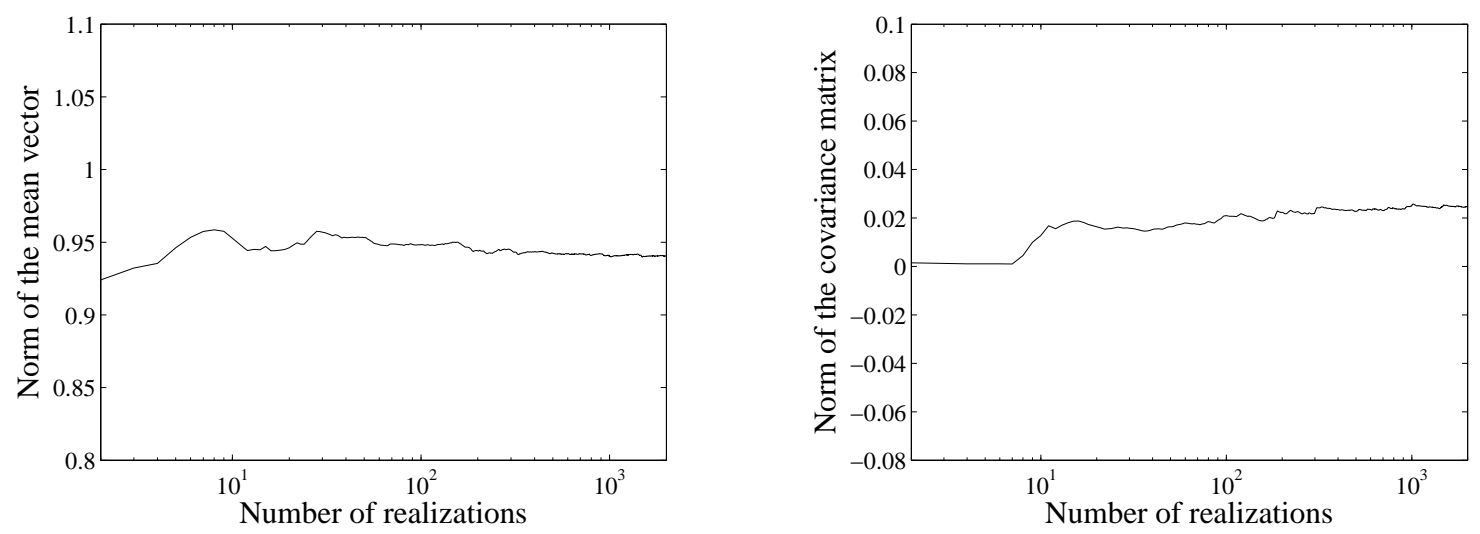

Figure 7: Convergence analysis: Euclidean norm of the statistical estimate of $\underline{\mathbf{h}}^{(4)}$ (left) and Frobenius norm of the statistical estimate of $\left[C_{\mathbf{H}^{(4)}}\right]$ (right).

It is seen that a reasonable convergence of the statistical estimates is reached for 1000 samples, so that we set $N_{\text {data }}=1000$. Optimization problem (29) is solved using the methodology introduced in Section 2.4, realizations of random vector $\boldsymbol{\eta}^{\text {pce }}$ being generated considering 10000 independent realizations of the Gaussian germ. For $N_{\text {ord }}=3$, one has $\epsilon_{1}^{p c e}(3)=0.4244, \epsilon_{2}^{p c e}(3)=0.4076, \epsilon_{3}^{p c e}(3)=0.4337$, $\epsilon_{4}^{p c e}(3)=0.2621$ and thus, $\operatorname{Conv}(3)=0.3819$. For $j=\{1, \ldots, 4\}$ and $N_{\text {ord }}=3$, the probability density functions $e \mapsto p_{\eta_{j}^{\text {sim }}}(e)$ and $e \mapsto p_{\eta_{j}^{\text {pce }}}(e)$ are plotted in Figs. 8 and 9 . It is seen that there is a good match between the reference and the chaos expansion-based (first-order marginal) distributions. Consequently, a third-order chaos expansion is used hereafter.

\subsection{Inverse identification}

This section deals with a methodology for the inverse identification of the probabilistic model presented in Section 2. The strategy is first introduced in Section 4.2.1. Each substep is then separately reviewed and illustrated. A few general comments are finally provided.

\subsubsection{Overall strategy.}

Let $\mathbf{W}^{\text {opt }}$ be the response random vector obtained by solving the SBVP using numerical Monte-Carlo simulations. Since the inverse identification is carried out on a limited set of data and solving a boundary value problem, we assume that the deterministic parameterizing fields are independent of $\mathbf{x}$ and that all the spatial correlation lengths of all the germs are equal. It is worth noticing that when full-field 

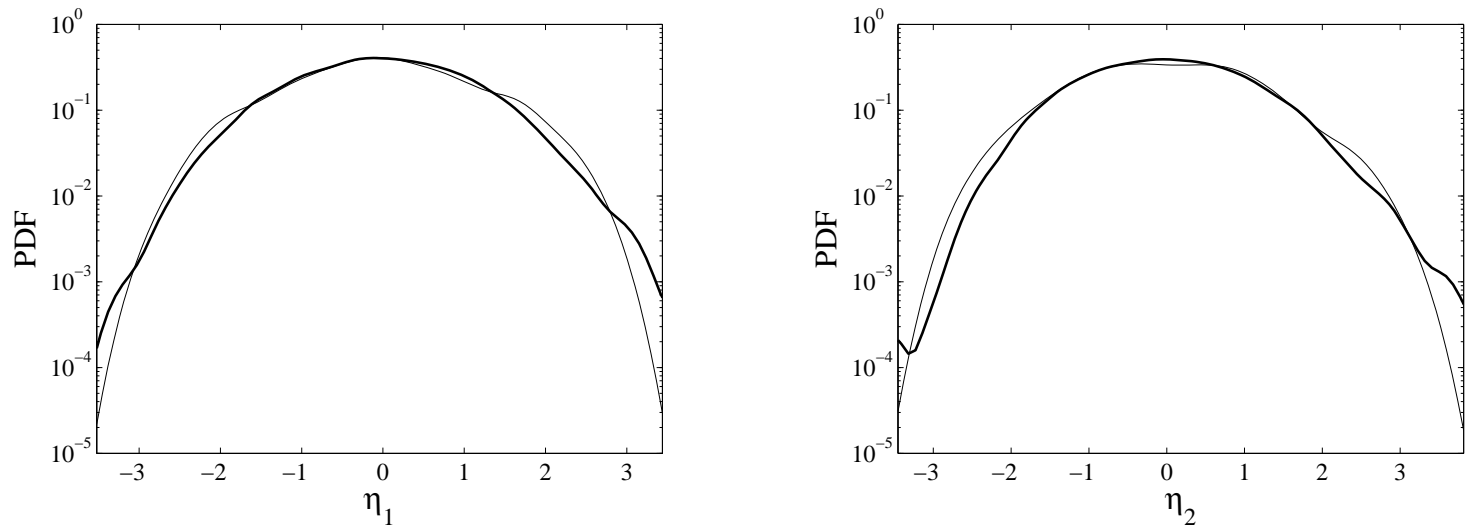

Figure 8: Comparison between the graphs (in semi-log scale) of the probability density functions $e \mapsto$ $p_{\eta_{j}^{\text {sim }}}(e)$ (thin solid line) and $e \mapsto p_{\eta_{j}^{p c e}}(e)$ (thick solid line), for $j=1$ (left) and $j=2$ (right).
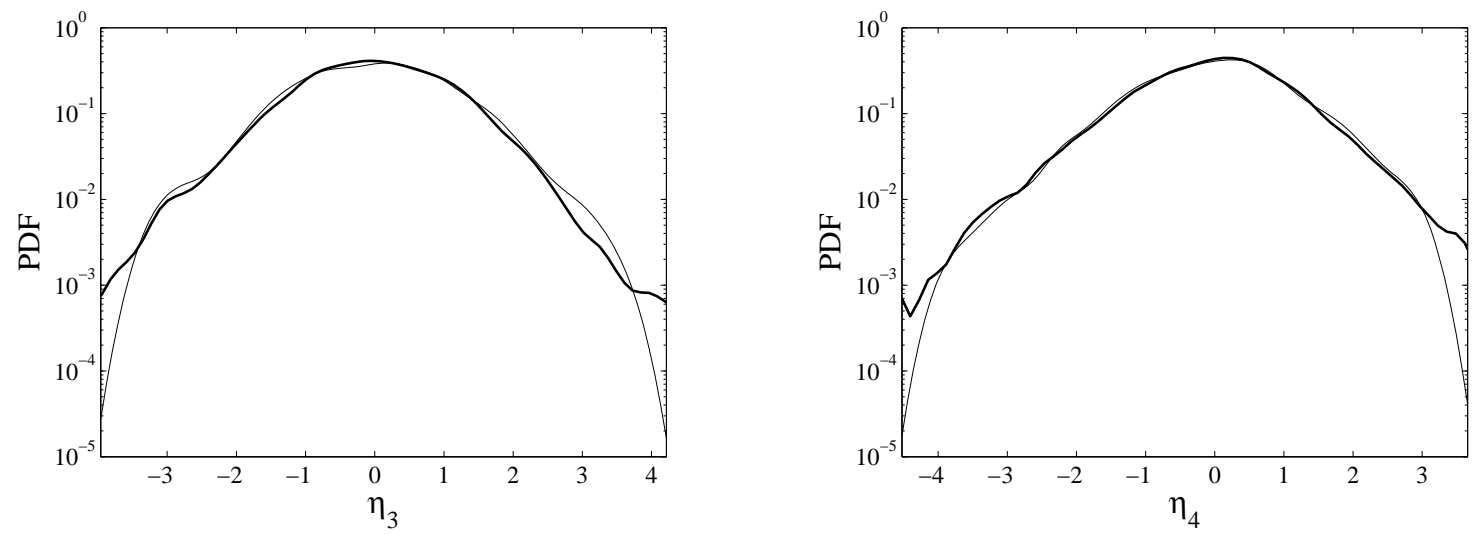

Figure 9: Comparison between the graphs (in semi-log scale) of the probability density functions $e \mapsto$ $p_{\eta_{j}^{s i m}}(e)$ (thin solid line) and $e \mapsto p_{\eta_{j}^{p c e}}(e)$ (thick solid line), for $j=3$ (left) and $j=4$ (right).

measurements are available, one may reasonably introduce nonconstant fields. From Section 2, one has $\mathbf{W}^{o p t}=\mathbf{W}^{o p t}\left(\left[\underline{L}^{o p t}\right], \alpha^{o p t}, \boldsymbol{\tau}^{o p t}, L_{c}^{o p t}\right)$, where superscript opt makes reference to parameters to be identified. The admissible space $\mathcal{C}^{\text {opt }}$ for the inverse identification can then be written as:

$$
\mathcal{C}^{\text {opt }}=\mathbb{M}_{n}^{+}(\mathbb{R}) \times \mathbb{R}_{*}^{+} \times \mathbb{R}^{+6} \times \mathbb{R}^{+} .
$$

Clearly, solving an inverse problem over such an admissible space is a very challenging task and may be computationally intractable in practice. Thus, we propose to proceed sequentially as follows:

- Step 1: sequential identification of a prior solution.

- a. Identification of the mean model, without statistical fluctuations. 
- b. Identification of the parameter $\alpha$ and of the spatial correlation lengths, without constraining the eigenvalues.

- c. Identification of the parameter $\tau$.

- Step 2: refinement of all the parameters in the neighborhood of the prior solution.

The first three substeps are now reviewed below.

\subsubsection{Step 1.a. Identification of a prior mean model.}

Let $\mathbf{w}^{\text {opt }}$ be the vector corresponding to the (observed) solution of the SBVP without fluctuations, that is, $\mathbf{w}^{o p t}=\mathbf{W}^{o p t}\left(\left[\underline{L}^{o p t}\right], \cdot, \cdot, \cdot\right)$. Let $\left[\underline{L}^{o p t}\right] \mapsto \mathcal{J}_{1}\left(\left[\underline{L}^{o p t}\right]\right)$ be the cost function defined as:

$$
\mathcal{J}_{1}\left(\left[\underline{L}^{o p t}\right]\right)=\left\|\underline{\mathbf{w}}^{\text {exp }}-\mathbf{w}^{o p t}\right\|_{\mathrm{F}}^{2},
$$

where $\underline{\mathbf{w}}^{e x p}=\mathrm{E}\left\{\mathbf{W}^{e x p}\right\}$. A first strategy for identifying a prior mean model would consist in solving the following optimization problem:

$$
\left[\underline{\widehat{L}}^{\text {prior }}\right]=\underset{\mathbb{M}_{n}^{+}(\mathbb{R})}{\arg \min } \mathcal{J}_{1}\left(\left[\underline{L}^{\text {opt }}\right]\right) .
$$

It is seen that Eq. (58) has to be solved over the entire interior of the positive-semidefinite cone. While the use of semidefinite programming algorithms allows solving such problems, it is worth noticing that (i) the obtained result may be highly questionable, taking into account the lack of constraints and experimental information, and that (ii) trying to identify a strongly anisotropic mean model from limited data is clearly meaningless. Subsequently, the prior mean model may be rather assumed to belong to a given class of material symmetry, reducing by the way the dimension of the optimization problem. For this purpose, available microstructural information may be used in order to infer about that class, which is here taken as $\mathcal{E}^{\mathrm{TI}}$ in view of Eq. (37). A prior mean model can then be identified solving:

$$
\left[\underline{\widehat{L}}^{\text {prior }}\right]=\underset{\mathcal{E}^{T I}}{\arg \min } \mathcal{J}_{1}\left(\left[\underline{L}^{\text {opt }}\right]\right) \text {. }
$$

Taking advantage of the well-known parametrization of $\mathcal{E}^{T I}$, optimization problem (59) is solved using a pattern search algorithm, initialized using a random perturbation of classical micromechanics-based estimates. The optimal prior mean value is given by (unit is [GPa]):

$$
\left[\underline{\underline{L}}^{\text {prior }}\right]=\left[\begin{array}{rrrrrr}
9.3247 & 0.6250 & 3.3581 & 0 & 0 & 0 \\
& 9.3247 & 3.3581 & 0 & 0 & 0 \\
& & 182.09 & 0 & 0 & 0 \\
& & & 14.2496 & 0 & 0 \\
& \text { Sym. } & & & 14.2496 & 0 \\
& & & & & 8.6997
\end{array}\right] .
$$

The matrix the components of which are the relative error (in \%) between the components of $[\underline{L}]$ (see Eq. (37)) and the ones of $\left[\underline{\hat{L}}^{\text {prior }}\right]$ is given below:

$$
\left[\epsilon_{r}\right]=\left[\begin{array}{rrrrrr}
7.71 & 15.93 & 13.35 & - & - & - \\
& 7.73 & 12.76 & - & - & - \\
& & 0.33 & - & - & - \\
& & & 1.54 & - & - \\
& \text { Sym. } & & & 1.69 & - \\
& & & & & 8.95
\end{array}\right] .
$$


It is seen that there is a relatively good agreement between the prior solution and the reference mean model, the maximum relative error being around $16 \%$.

\subsubsection{Step 1.b. Identification of prior values for $\alpha$ and $L$.}

The second step in the methodology consists in finding the best values of scalar parameters $\alpha^{\text {opt }}$ and $L_{c}^{o p t}$ in the admissible space $\mathbb{R}^{+} \times \mathbb{R}^{+}$, setting $\left[\underline{L}^{o p t}\right]=\left[\underline{\hat{L}}^{\text {prior }}\right]$ and $\boldsymbol{\tau}^{\text {opt }}=\mathbf{0}$. Consequently, one has $\mathbf{W}^{o p t}=\mathbf{W}^{o p t}\left(\left[\widehat{\underline{L}}^{p r i o r}\right], \alpha^{o p t}, \mathbf{0}, L_{c}^{o p t}\right)$. Let $\left(\alpha^{o p t}, L_{c}^{o p t}\right) \mapsto \mathcal{J}_{2}\left(\alpha^{o p t}, L_{c}^{o p t}\right)$ be the cost function defined as:

$$
\mathcal{J}_{2}\left(\alpha^{o p t}, L_{c}^{o p t}\right)=\left(1-p_{\mathcal{J}_{2}}\right)\left\|\underline{\mathbf{w}}^{e x p}-\underline{\mathbf{w}}^{o p t}\right\|_{\mathrm{F}}^{2}+p_{\mathcal{J}_{2}}\left\|\boldsymbol{\sigma}^{e x p}-\boldsymbol{\sigma}^{o p t}\right\|_{\mathrm{F}}^{2},
$$

in which $p_{\mathcal{J}_{2}} \in[0,1]$ is a free parameter and $\underline{\mathbf{w}}^{\text {opt }}=\mathrm{E}\left\{\mathbf{W}^{\text {opt }}\right\}$. Vectors $\boldsymbol{\sigma}^{\text {exp }}$ and $\boldsymbol{\sigma}^{\text {opt }}$ are such that components $\boldsymbol{\sigma}^{\text {exp }}{ }_{i}$ and $\boldsymbol{\sigma}^{\text {opt }}{ }_{i}$ are the standard deviations of components $\mathbf{W}^{\text {exp }}{ }_{i}$ and $\mathbf{W}^{\text {opt }}{ }_{i}$ respectively. Estimates of $\underline{\mathbf{w}}^{o p t}$ and $\boldsymbol{\sigma}^{o p t}$ are obtained from a set of 500 simulated realizations. Parameters $\alpha$ and $L_{c}$ are identified solving the following optimization problem:

$$
\left(\widehat{\alpha}^{\text {prior }}, \widehat{L}_{c}^{\text {prior }}\right)=\underset{\mathbb{R}^{+} \times \mathbb{R}^{+}}{\arg \min } \mathcal{J}_{2}\left(\alpha^{o p t}, L_{c}^{o p t}\right) .
$$

Eq. (63) is solved using a trial method, that is to say, by computing the value of the cost function $\mathcal{J}_{2}$ for selected values of parameters $\alpha^{\text {opt }}$ and $L_{c}^{\text {opt }}$. Following Section 2.8, we considered $\alpha^{\text {opt }} \in$ $\{20,60,100,140,180\}$. Furthermore, the spatial correlation length can be reasonably assumed to be less than the size the domain $\Omega$, so that we set $L_{c}^{o p t} \in\{0.1,0.2,0.3,0.4,0.5,0.6\}$. Finally, two values of parameter $p_{\mathcal{J}_{2}}$ (namely, $p_{\mathcal{J}_{2}}=0.5$ and $p_{\mathcal{J}_{2}}=0.8$ ) were tested and yielded very similar results. For $p_{\mathcal{J}_{2}}=0.5$, the values of cost function $\mathcal{J}_{2}$ (normalized by a factor $10^{-18}$ ) for all the trial values are reported in a matrix form in Eq. (64) (a raw -resp. a column - corresponds to a given value of $\alpha^{o p t}$-resp. to a given value of $\left.L_{c}^{o p t}-\right)$.

$$
\left[\mathcal{J}_{2}\right]=\left[\begin{array}{llllll}
0.8137 & 2.3540 & 2.5909 & 3.7354 & 4.2137 & 4.1469 \\
0.3159 & 0.0911 & 0.0495 & 0.0618 & 0.0844 & 0.0822 \\
0.9326 & 0.4661 & 0.3860 & 0.2327 & 0.1947 & 0.2033 \\
1.4239 & 0.8945 & 0.8037 & 0.6003 & 0.5444 & 0.5565 \\
1.8020 & 1.2572 & 1.1634 & 0.9420 & 0.8796 & 0.8929
\end{array}\right] .
$$

The corresponding graph $\left(\alpha^{o p t}, L_{c}^{o p t}\right) \mapsto \mathcal{J}_{2}\left(\alpha^{o p t}, L_{c}^{o p t}\right)$ is shown in Fig. 10. It is readily seen that the minimum of the cost function is obtained for $\widehat{\alpha}^{\text {prior }}=60$ and ${\widehat{L_{c}}}^{\text {prior }}=0.3$, that is to say, for the reference values of the parameters.

\subsubsection{Step 1.c. Identification of a prior value for vector $\tau$.}

Let us now consider $\mathbf{W}^{\text {opt }}=\mathbf{W}^{\text {opt }}\left(\left[\underline{\underline{L}}^{\text {prior }}\right], \widehat{\alpha}^{\text {prior }}, \boldsymbol{\tau}^{\text {opt }},{\widehat{L_{c}}}^{\text {prior }}\right)$, where $\boldsymbol{\tau}^{\text {opt }}$ is given by:

$$
\boldsymbol{\tau}^{o p t}=\left(\tau_{1}^{o p t}, \tau_{2}^{o p t}, \tau_{3}^{o p t}, \tau_{4}^{o p t}, \tau_{5}^{o p t}, \tau_{6}^{o p t}\right) .
$$

Following the same approach as in Section 4.2.3, let $\tau^{\text {opt }} \mapsto \mathcal{J}_{3}\left(\boldsymbol{\tau}^{\text {opt }}\right)$ be the cost function defined as:

$$
\mathcal{J}_{3}\left(\boldsymbol{\tau}^{o p t}\right)=\left(1-p_{\mathcal{J}_{3}}\right)\left\|\underline{\mathbf{w}}^{\text {exp }}-\underline{\mathbf{w}}^{o p t}\right\|_{\mathrm{F}}^{2}+p_{\mathcal{J}_{3}}\left\|\boldsymbol{\sigma}^{e x p}-\boldsymbol{\sigma}^{o p t}\right\|_{\mathrm{F}}^{2},
$$




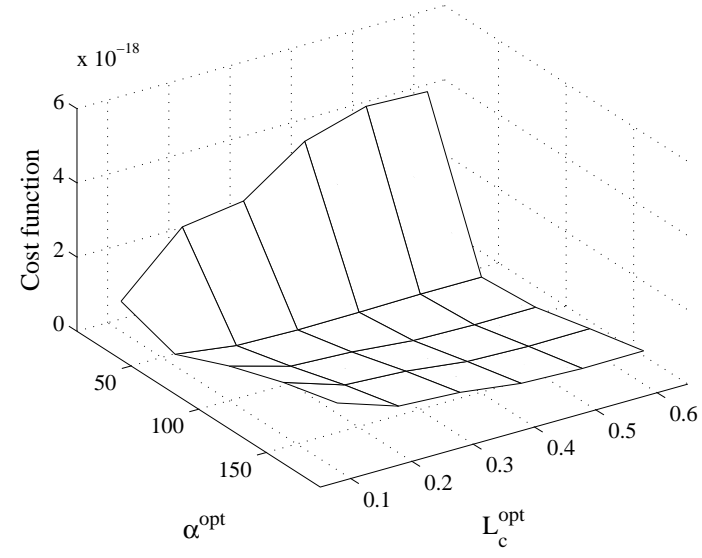

Figure 10: Plot of the cost function $\left(\alpha^{o p t}, L_{c}^{o p t}\right) \mapsto \mathcal{J}_{2}\left(\alpha^{o p t}, L_{c}^{o p t}\right)$ for $p_{\mathcal{J}_{2}}=0.5$.

in which $p_{\mathcal{J}_{3}} \in[0,1]$. A prior estimate of $\tau$ can be obtained solving:

$$
\widehat{\boldsymbol{\tau}}^{\text {prior }}=\underset{\mathbb{R}^{+6}}{\arg \min } \mathcal{J}_{3}\left(\boldsymbol{\tau}^{\text {opt }}\right) .
$$

Taking into account the large range of possible values for all the parameters $\tau_{i}^{\text {opt }}, i=1, \ldots, 6$, Eq. (67) can be solved using a trial method for which the values are selected using a logarithmic scale. One may set, for instance, $\tau_{i}^{\text {opt }} \in\left\{1,10^{1}, 10^{2}, 10^{3}\right\}$. Allowing parameter $\tau_{i}^{\text {opt }}$ to take $k$ different values then implies computing the cost function (66) $k^{6}$ times (that are, 4096 times for $k=4$ for instance). Following the discussion provided in Section 3, one may alternatively consider identifying parameter $\tau^{\text {opt }}$ with reference to the distance to a given material symmetry class. While such a strategy is clearly restricted to random media the realizations of which may still exhibit strong symmetries (in other words, the distance of each realization to the relevant symmetry class is bounded), such as unidirectional composites, it benefits from a minimal parametrization, since the resulting optimization problem may be formulated using a single unknown parameter. Since $\left[\widehat{\widehat{L}}^{\text {prior }}\right] \in \mathcal{E}^{\mathrm{TI}}$ and following the second approach, one may reasonably consider $\widehat{\boldsymbol{\tau}}^{\text {prior }}=\left(\widehat{\tau}^{\text {prior }}, \widehat{\tau}^{\text {prior }}, 0, \widehat{\tau}^{\text {prior }}, \widehat{\tau}^{\text {prior }}, 0\right)$, in which $\widehat{\tau}^{\text {prior }}$ is the solution of the following optimization problem:

$$
\widehat{\tau}^{\text {prior }}=\underset{\mathbb{R}^{+}}{\arg \min } \mathcal{J}_{3}\left(\left(\tau^{o p t}, \tau^{o p t}, 0, \tau^{o p t}, \tau^{o p t}, 0\right)\right) .
$$

Eq. (68) is solved by the trial method for $\tau^{\text {opt }} \in\{1,10,100,200,300,500,600\}$. The plot of function $\tau^{o p t} \mapsto \mathcal{J}_{3}\left(\left(\tau^{o p t}, \tau^{o p t}, 0, \tau^{o p t}, \tau^{o p t}, 0\right)\right.$ ) is reported in Fig. 11 (for $p_{\mathcal{J}_{3}}=0.5$ ).

It is seen that the minimal value is obtained for $\widehat{\tau}^{\text {prior }}=200$. 


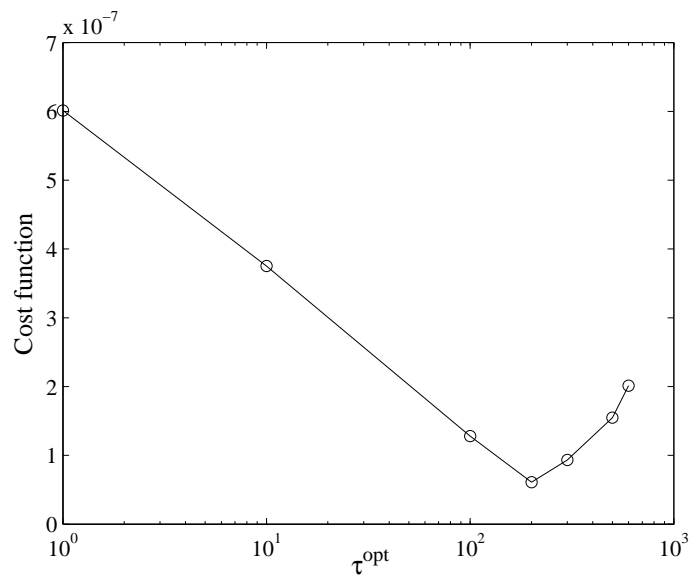

Figure 11: Plot of the cost function $\tau^{\text {opt }} \mapsto \mathcal{J}_{3}\left(\left(\tau^{o p t}, \tau^{o p t}, 0, \tau^{o p t}, \tau^{o p t}, 0\right)\right)$ for $p_{\mathcal{J}_{3}}=0.5$.

\subsubsection{Summary and general comments about inverse identification.}

Based on the previous sections, prior estimates of the parameters are then given by:

$$
\begin{aligned}
& {\left[\underline{\underline{L}}^{\text {prior }}\right]=\left[\begin{array}{rrrrrr}
9.3247 & 0.6250 & 3.3581 & 0 & 0 & 0 \\
& 9.3247 & 3.3581 & 0 & 0 & 0 \\
& & 182.09 & 0 & 0 & 0 \\
& & & 14.2496 & 0 & 0 \\
& \text { Sym. } & & & 14.2496 & 0 \\
& & & & & 8.6997
\end{array}\right] \text {, }} \\
& {\widehat{L_{c}}}^{\text {prior }}=0.3 \text {, } \\
& \widehat{\alpha}^{\text {prior }}=60, \\
& \widehat{\boldsymbol{\tau}}^{\text {prior }}=(200,200,0,200,200,0) .
\end{aligned}
$$

The perfect agreement of the prior solutions ${\widehat{L_{c}}}^{\text {prior }}, \widehat{\alpha}^{\text {prior }}$ and $\widehat{\tau}^{\text {prior }}$ with the reference values of the parameters is obviously due to the fact that the latter belong to the sets of trial values. Since it may not be the case in general, one may then have recourse to a refinement in the neighborhood of the prior estimates. Note that this second step, which has not been illustrated here, does not introduce any additional difficulty and that the resulting increase of computational cost may not be a critical issue, since in particular, solving by a trial method is well suited for parallelization.

Three conclusions can further be drawn and are worth taking into account while addressing the inverse identification. First of all, it is readily seen, from the parametric study performed in this research, that parameters to be identified generally induce asymptotic behaviors that are well-known and from which some bounds (to be used for constraining the optimization solver) can be deduced. For instance, setting values of $\alpha$ larger than 100 generally implies a small level of overall fluctuations, while small values of this parameter yields larger statistical fluctuations. Consequently, it is likely that for mesoscopic domain 
which are sufficiently large (but much smaller than the RVE size, in order to be consistent with the modeling of apparent properties), parameter $\alpha$ may be sought within, say 20 and 100 . The same way of reasoning holds for the spatial correlation lengths, since local fluctuations are often (but not always) induced by the local random topology, so that initial guesses for parameters $\left\{L_{k}^{i \ell}\right\}_{k, i, \ell}$ may be chosen in accordance with some characteristic lengths of the local topology. Note also that most engineered or even biological materials exhibits some material symmetries (at least, at the macroscale), so that the form of vector $\tau$ may be reasonably inferred as well. In other words, although the proposed application does not make reference to such information (since it relies on a simulated database), one should always take into account as much information as possible in order to reduce the computational cost associated with the inverse identification procedure.

Secondly, the model parameter identification is arguably made easier by the fact that modifications of each parameter do have a significant impact on the realizations of the apparent elasticity tensor random field (which may be more or less anisotropic, etc.) and therefore, on the solution displacement field, so that the proposed objective functions exhibit rather large fluctuations over the admissible spaces (note that this may not be the case for all applications though).

Finally, it should be pointed out that no matter the numerical strategy that is used (least square method, maximum likelihood principle, etc.), the inverse identification basically consists in finding the closest approximation in the proposed class of non-Gaussian positive-definite matrix-valued random fields. Thus, while the reduced parametrization makes this class especially suitable for inverse identification (as well as for stochastic modeling on large-scale systems), it may introduce some discrepancy between the experimental, true random field and its approximation. However, this potential difficulty can be circumvented by considering a more general identification strategy, in which the proposed class can be used as a class of prior algebraic stochastic models to be combined, within an updating procedure, to functional (chaos) representations (with final random coefficients). Such a methodology, which has been proposed and successfully applied in [27], underlines the fact that the information used in the MaxEnt approach (while defining the class of prior algebraic models) must be as rich as possible in order to make the updating procedure reasonably feasible (and especially, when one is concerned with high dimension modeling and/or makes use of the Bayesian method). From that point of view, it is believed that information related to material symmetries is of primal importance in many practical situations.

\section{CONCLUSION}

In this paper, we have proposed the construction of a class of prior stochastic models for non-Gaussian positive-definite matrix-valued random fields. In particular, this class exhibits a larger number of parameters than the other classes previously derived within a nonparametric framework. Making use of a coordinate-free characterization of material symmetry classes, it is then shown that the probabilistic model may allow prescribing higher statistical fluctuations in given directions. Consequently, such stochastic fields turn out to be especially suitable for the fundamental issue of inverse experimental identification under material symmetry uncertainties. It it worth noticing that they can also be used as stochastic fields for the development of computational multi-scale approaches in which the underlying randomness arising from fine scale features has to be taken into account at a coarse scale. We finally present and illustrate a possible strategy for inverse identification, relying on the sequential solving of least-square optimization problems. While the methodology is exemplified in the context of the mesoscale modeling of an elasticity tensor random field, the proposed class is also well suited for other applications involving non-Gaussian positive-definite matrix-valued random fields, such as the modeling of the flow through random porous 
media characterized by a permeability tensor random field.

\section{ACKNOWLEDGEMENTS}

This research was funded by the French Research Agency (Agence Nationale de la Recherche) under TYCHE contract ANR-2010-BLAN-0904.

\section{References}

[1] V. Arsigny, P. Fillard, X. Pennec, and N. Ayache. Log-Euclidean metrics for fast and simple calculus on diffusion tensors. Magnetic Resonance in Medicine, 56:411-421, 2006.

[2] A. Bóna, I. Bucataru, and M. A. Slawinski. Coordinate-free characterization of the symmetry classes of elasticity tensors. Journal of Elasticity, 87:109-132, 2007.

[3] J.T. Browaeys and S. Chevrot. Decomposition of the elastic tensor and geophysical applications. Geophys. J. Int., 159:667-678, 2004.

[4] S. Das and R. Ghanem. A bounded random matrix approach for stochastic upscaling. Multiscale Modeling ES Simulation, 8(1):296-325, 2009.

[5] S. Das, R. Ghanem, and J. Spall. Asymptotic sampling distribution for polynomial chaos representation of data: A maximum-entropy and fisher information approach. SIAM Journal on Scientific Computing, 30(5):2207-2234, 2008.

[6] C. Desceliers, R. Ghanem, and C. Soize. Maximum likelihood estimation of stochastic chaos representations from experimental data. International Journal of Numerical Methods in Engineering, 66(6):978-1001, 2006.

[7] R. Ghanem and P. Spanos. Stochastic finite elements: a Spectral Approach. Springer, New-York, 1991.

[8] J. Guilleminot and C. Soize. A stochastic model for elasticity tensors with uncertain material symmetries. International Journal of Solids and Structures, 47(22-23):3121-3130, 2010.

[9] S. Hazanov and C. Huet. Order relationships for boundary conditions effect in heterogeneous bodies smaller than the representative volume. Journal of the Mechanics and Physics of Solids, 42(12):19952011, 1994.

[10] C. Huet. Application of variational concepts to size effects in elastic heterogeneous bodies. Journal of the Mechanics and Physics of Solids, 38(6):813-841, 1990.

[11] E. T. Jaynes. Information theory and statistical mechanics. Physical Review, 106(4):620-630, 1957.

[12] E. T. Jaynes. Information theory and statistical mechanics. Physical Review, 108(2):171-190, 1957.

[13] M. P. Mignolet and C. Soize. Nonparametric stochastic modeling of linear systems with prescribed variance of several natural frequencies. Probabilistic Engineering Mechanics, 23:267-278, 2008. 
[14] M. Moakher. On the averaging of symmetric positivedefinite tensors. Journal of Elasticity, 82:273$296,2006$.

[15] M. Moakher and A. N. Norris. The closest elastic tensor of arbitrary symmetry to an elasticity tensor of lower symmetry. Journal of Elasticity, 85:215-263, 2006.

[16] R. M. Neal. Slice sampling. Annals of Statistics, 31(3):705-767, 2003.

[17] S. Nemat-Nasser and M. Hori. Micromechanics: Overall Properties of Heterogeneous Materials. Chapman \& Hall-CRC-Taylor \& Francis, North-Holland, 1993.

[18] M. Ostoja-Starzewski. Microstructural Randomness and Scaling in Mechanics of Materials. Chapman \& Hall-CRC-Taylor \& Francis, 2008.

[19] M. Rosenblatt. Remark on a multivariate transformation. The Annals of Mathematical Statistics, 23(3):470472, 1952.

[20] K. Sab. On the homogenization and the simulation of random materials. European Journal of Mechanics A/Solids, 11(5):585-607, 1992.

[21] R.J. Serfling. Approximation Theorems of Mathematical Statistics. John Wiley \& Sons, 1980.

[22] C. E. Shannon. A mathematical theory of communication. Bell System Technical Journal, 27:379423/623-659, 1948.

[23] C. Soize. A nonparametric model of random uncertainties on reduced matrix model in structural dynamics. Probabilistic Engineering Mechanics, 15(3):277-294, 2000.

[24] C. Soize. Non-gaussian positive-definite matrix-valued random fields for elliptic stochastic partial differential operators. Computer Methods in Applied Mechanics and Engineering, 195:26-64, 2006.

[25] C. Soize. Tensor-valued random fields for meso-scale stochastic model of anisotropic elastic microstructure and probabilistic analysis of representative volume element size. Probabilistic Engineering Mechanics, 23:307-323, 2008.

[26] C. Soize. Generalized probabilistic approach of uncertainties in computational dynamics using random matrices and polynomial chaos decompositions. International Journal of Numerical Methods in Engineering, 81(8):939-970, 2010.

[27] C. Soize. Identification of high-dimension polynomial chaos expansions with random coefficients for non-gaussian tensor-valued random fields using partial and limited experimental data. Computer Methods in Applied Mechanics and Engineering, 199:2150-2164, 2010.

[28] C. Soize, E. Capiez-Lernout, J.-F. Durand, C. Fernandez, and L. Gagliardini. Probabilistic model identification of uncertainties in computational models for dynamical systems and experimental validation. Computer Methods in Applied Mechanics and Engineering, 198(1):150-163, 2008.

[29] C. Soize and R. Ghanem. Physical systems with random uncertainties: chaos representations with arbitrary probability measure. SIAM Journal on Scientific Computing, 26:395-410, 2004.

[30] Q.A. Ta, D. Clouteau, and R. Cottereau. Modeling of random anisotropic elastic media and impact on wave propagation. European Journal of Computational Mechanics, 19(1-2-3):241-253, 2010. 
[31] N. Wiener. The homogeneous chaos. American Journal of Mathematics, 60(4):897-936, 1938.

[32] X. Yina, W. Chen, A. To, C. McVeigh, and W. K. Liu. Statistical volume element method for predicting microstructure-constitutive property relations. Computer Methods in Applied Mechanics and Engineering, 197(43-44):3516-3529, 2008. 